Journal for ImmunoTherapy of Cancer

\section{Relaxin-expressing oncolytic adenovirus induces remodeling of physical and immunological aspects of cold tumor to potentiate PD-1 blockade}

To cite: Jung B-K, Ko HY, Kang $\mathrm{H}$, et al. Relaxinexpressing oncolytic adenovirus induces remodeling of physical and immunological aspects of cold tumor to potentiate PD-1 blockade. Journal for ImmunoTherapy of Cancer 2020;8:e000763. doi:10.1136/ jitc-2020-000763

- Additional material is published online only. To view, please visit the journal online (http://dx.doi.org/10.1136/jitc2020-000763).

B-KJ and HYK contributed equally.

JSK and C-OY contributed equally.

Accepted 24 June 2020

Check for updates

(c) Author(s) (or their employer(s)) 2020. Re-use permitted under CC BY. Published by BMJ.

For numbered affiliations see end of article.

Correspondence to

Dr Chae-Ok Yun;

chaeok@hanyang.ac.kr

Dr Jin Su Kim; kjs@kirams.re.kr

\section{ABSTRACT}

Background Currently, several antibody (Ab)-based therapies have shown excellent therapeutic effects in the clinic. Nonetheless, Ab penetration into tumor tissues is limited due to abnormal vasculature, tumor interstitial pressure, and excessive extracellular matrix (ECM) accumulation, thus demanding novel strategies to overcome these barriers.

Methods The intratumoral distribution of therapeutic Abs were detected by fluorescence microscopy or positron emission tomography in both human gastric xenograft and syngeneic pancreatic hamster tumor models. The antitumor efficacy by combination of oncolytic adenovirus (Ad), which coexpresses relaxin (RLX), interleukin (IL)-12, and granulocyte macrophage colony-stimulating factor (GM-CSF) (oAd/IL12/ GM-RLX) and antibody against the programmed cell death protein $1(\alpha P D-1)$ was examined in hamster subcutaneous and orthotopic pancreatic tumor models. The immunological aspects of these combination therapy regimen were assessed by flow cytometry or immunohistochemistry in subcutaneous hamster tumor models.

Results Relaxin-expressing oncolytic Ad effectively degraded tumor ECM and enhanced the tumor penetration of trastuzumab in comparison with trastuzumab monotherapy. Based on these results, an oAd/IL12/GM-RLX was used to enhance the potency of immune checkpoint blockade. The combination of the oAd/IL12/GM-RLX and $\alpha \mathrm{PD}-1$ promoted a concomitant degradation of the tumor ECM and amelioration of the immunosuppressive tumor niches, ultimately enhanced intratumoral infiltration of both $\alpha \mathrm{PD}-1$ and activated T cells. Of note, the combination therapy was able to elicit a potent and durable antitumor immune response against cold tumors that were refractory to immune checkpoint inhibitor monotherapy.

Conclusions Our findings are the first to demonstrate that expression of four genes (IL-12p35, IL-12p40, GM-CSF, and $R L X)$ mediated by a single oncolytic Ad vector can promote remodeling of both physical and immunological aspects of the tumor niches to overcome the major limitations of $A b$-based therapies that have emerged in recent clinical trials.

\section{BACKGROUND}

Antibody (Ab)-based cancer therapy has been one of the most effective strategies for treating patients with tumors. More than 20 products have been approved by the US Food and Drug Administration for cancer, and other products are investigated in clinical trials. ${ }^{1}$ The mechanisms of tumor cell killing by therapeutic Abs include a direct action of the $\mathrm{Ab}$ and immune response-mediated cell killing mechanisms. ${ }^{2}$

Several tumor microenvironmental factors, which include an abnormal vasculature, elevated interstitial pressure, high cell density, and dense extracellular matrix (ECM), function as the primary barriers against drug penetration. ${ }^{3}$ Tumor-derived ECM, which consists of a dense network of various collagens, fibronectins, and proteoglycans, inhibits intratumoral penetration and dispersion of therapeutics. ${ }^{4}$ Thus, determining strategies to breach the ECM is an important goal.

Preferential transfer of a gene encoding an ECM-degrading protein to tumor by viral vectors could induce durable ECM degradation. Among several vectors in development for cancer therapy, oncolytic adenovirus (Ad) is particularly promising due to proven safety record of Ad in numerous clinical trials, preferential cytolytic activity against tumor, no risk of insertional mutagenesis, high gene transfer efficacy, and cancer-specific and transient therapeutic gene expression that is amplified with each replication cycle and secondary infection of adjacent cancer cells. ${ }^{5}$ oAd without ECM-degrading transgene cannot be efficiently dispersed away from the injection site in tumor tissues due to dense tumor ECM; thus, we have employed relaxin (RLX) as one of the therapeutic transgene in present report due to its ability to inhibit the de novo ECM synthesis and to decompose the overexpressed ECM components in diseases with aberrant fibrosis (eg, keloid and desmoplasia in tumor), as well as improving viral 
dispersion in tumor tissues. ${ }^{6}$ Further, we hypothesized that ECM degradation by RLX-expressing oAd will also aid the intratumoral penetration and dispersion of therapeutic Abs.

Among therapeutic Abs, immune checkpoint inhibitors (ICIs), such as antibody against the programmed cell death protein 1 ( $\alpha \mathrm{PD}-1)$ or anti-cytotoxic T-lymphocyte antigen $4 \mathrm{Abs}$, have been extensively evaluated in various phases of clinical development for their ability to elicit potent antitumor immune response. ${ }^{7}$ Although ICI treatments can lead to durable responses against multiple types of cancers in a subset of patients, they are ineffective in patients harboring 'cold' tumors in which the level of immune cell infiltration is low. ${ }^{8}$ In this regard, the development of rationally designed combination therapy strategies is necessary to improve the effect of ICIs by facilitating $\mathrm{CD}^{+}$and $\mathrm{CD}^{+}{ }^{+}$T-cell recruitment and activation in the tumor microenvironment. ${ }^{10}$ Oncolytic viruses encoding tumor antigens, cytokines, and/or costimulatory molecules could be a promising means of enhancing ICI activity in 'cold' tumors, with numerous reports demonstrating that oncolytic viruses boost antitumor immune responses. ${ }^{11}$

In this study, we used RLX-expressing and immunostimulatory cytokine-expressing oncolytic Ads to enhance the penetration and immune response mediated by an ICI against immunosuppressive pancreatic tumors, which are extremely resistant toward ICI due to desmoplasia, restricting intratumoral infiltration of T cells. ${ }^{12}$ First, we demonstrate that a relaxin-expressing oncolytic adenovirus (oAd/RLX) increases the intratumoral distribution and penetration of trastuzumab by promoting the degradation of ECM in a gastric cancer xenograft model. Building on these initial results, we combined $\alpha$ PD- 1 with an immune stimulatory and ECM degrading oncolytic Ad, which coexpresses interleukin (IL)-12, granulocyte macrophage colony-stimulating factor (GM-CSF), and RLX, to elicit potent and durable antitumor immune responses against pancreatic cancer. Importantly, triple therapeutic gene expression mediated by oncolytic Ad enhances the intratumoral distribution of $\alpha \mathrm{PD}-1 \mathrm{Ab}$ and concomitantly boosts the antitumoral immune response through improved intratumoral infiltration of activated $\mathrm{T}$ cells, demonstrating that oncolytic Ad-mediated remodeling of both physical and immunological aspects of the tumor microenvironment is a promising strategy for enhancing the potency of ICI against poorly immunological solid tumors.

\section{MATERIALS AND METHODS Cell lines and cell culture}

The human gastric cancer cell line NCI-N87 was cultured in RPMI-1640 medium (GIBCO-BRL, Grand Island, New York, USA). The hamster pancreatic carcinoma cell line HaP-T1 and luciferase-expressing HP-1 (HP-1/Fluc) were cultured in Dulbecco's modified Eagle's medium (GIBCO-BRL). All growth medium was supplemented with $10 \%$ fetal bovine serum (FBS; GIBCO-BRL), $1 \%$ penicillin-streptomycin $(100 \mathrm{U} / \mathrm{mL})$. All cells were maintained at $37^{\circ} \mathrm{C}$ in a humidified atmosphere with $5 \%$ $\mathrm{CO}_{2}$. The NCI-N87 cell line was purchased from American Type Culture Collection (Manassas, Virginia, USA); HaP-T1 cell line was kindly provided by Dr Masato Abei (University of Tsukuba, Ibaraki, Japan); and HP-1/Fluc, which stably express firefly luciferase, was generated using a lentiviral vector, using the HP-1 cell line provided by Dr Masato Yamamoto (University of Minnesota, Minneapolis, Minnesota).

\section{Virus preparation}

The construction, generation, and characterization of oAd/RLX have been reported previously. ${ }^{13}$ To generate oAd/IL12/GM-RLX, we first constructed a pSP72-E3 Ad E3 shuttle vector expressing GM-CSF and RLX (pSP72-E3/GM-RLX). The pSP72-E3/GM-RLX vector was constructed by subcloning the GM-CSF-Internal ribosome entry site (IRES) PCR product into the previously generated pSP72-E3/RLX vector. ${ }^{13}$ The newly constructed pSP72-E3/GM-RLX shuttle vector was linearized with $X m n I$ digestion, and pAd- $\Delta \mathrm{B} 7 / \mathrm{IL}-12$, an Ad-based E1B/ E3-deleted total vector with a substitution in the retinoblastoma binding sites of E1A, which expresses IL-12 in the E1 region of $\mathrm{Ad}-\Delta \mathrm{B} 7,{ }^{14}$ was linearized with SpeI digestion. The linearized pSP72-E3/GM-RLX E3 shuttle vector was then cotransformed into Escherichia coli BJ5183, along with the SpeI-digested pAd- $\Delta$ B7/IL-12 for homologous recombination, resulting in the pAd- $\Delta \mathrm{B} 7 / \mathrm{IL}-12 /$ GM-CSF-IRES-RLX Ad vector. To produce the corresponding Ad, purified plasmids were digested with PacI and transfected into 293A cells, a human embryonic kidney cell line expressing the Ad E1 region, to generate oAd- $\Delta$ B 7/IL-12/GM-CSF-IRES-RLX

(oAd/IL12/ GM-RLX). All Ads were propagated in 293A cells and purified by $\mathrm{CsCl}$ gradient centrifugation. The number of viral particles (VPs) was determined by measuring the optical density at $260 \mathrm{~nm}$, for which an absorbance value of 1 is equivalent to $1.1 \times 10^{12} \mathrm{VP} / \mathrm{mL}$.

\section{Preparation of Alexa Fluor 488-conjugated Ab}

A solution (10 mM) of Alexa Fluor 488 (Invitrogen, Grand Island, New York, USA) was dissolved in dimethyl sulfoxide with $1 \%$ acetic acid. The solution was mixed with $5 \mathrm{mg}$ of Trastuzumab (TZB; Roche, Basel, Switzerland) or $\alpha$ PD-1 (clone RMP1-14; Bio X Cell, West Lebanon, New Hampshire, USA) in $250 \mu \mathrm{L}$ of $1 \mathrm{M}$ sodium bicarbonate solution, $\mathrm{pH} 8.5$ and allowed to stand for 1 hour at room temperature. The Alexa Fluor 488-conjugated $\mathrm{Ab}$ was purified with a size exclusion PD-10 column (GE Healthcare Bio-Sciences AB, Uppsala, Sweden). The number of Alexa Fluor 488 molecules conjugated per $\mathrm{Ab}$ was estimated by determining the Alexa Fluor 488 peak intensity distribution between the Ab-Alexa Fluor 488 conjugate and the free Alexa Fluor 488 eluted from the size-exclusion HPLC column (Waters Corporation, Milford, Massachusetts, USA). 
Assessment of trastuzumab distribution in tumor tissue

Nude mice were subcutaneously inoculated with $5 \times 10^{6}$ NCI-N87 cells. When the average tumor volume reached $200 \mathrm{~mm}^{3}$, tumor-bearing mice were intravenously administered with phosphate-buffered saline (PBS), Alexa 488-conjugated TZB (488-TZB; $150 \mu \mathrm{g}$ ), or oAd/RLX $\left(2.5 \times 10^{10} \mathrm{VP}\right)$ plus 488 -TZB $(150 \mu \mathrm{g})$. The first day of treatment was designated as day 0 . oAd/RLX was administered three times in total, whereas a single dose of 488TZB was administered. On the fifth day after the last administration, $1 \mathrm{mg}$ of rhodamine-lectin (rhodamine ricinus communis agglutinin I) was intravenously injected for visualization of blood vessels. Tumors were harvested with intact skin and flash-frozen using liquid nitrogen for subsequent sectioning and staining. Tumor sections were fixed with $4 \%$ paraformaldehyde for 10 min and mounted with Prolong Gold antifade reagent with 4,6-diamidino-2-phenyindole (DAPI) (Invitrogen, Carlsbad, California, USA).

\section{Acquisition and analysis of fluorescent images}

Imaging was performed with a $\times 10$ objective lens using a fluorescent microscope (IN Cell Analyzer, GE Healthcare, Waukesha, Wisconsin, USA) and equipped with mosaic stitching software (IN Cell developer toolbox, GE Healthcare). Three independent channels were obtained: DAPI for nuclei (blue), rhodamine for blood vessels (red), and Fluorescein isothiocyanate (FITC) for 488-TZB (green). The pixel size was $0.65 \mu \mathrm{m} /$ pixel.

Values were pooled together from 40 regions of each sample to represent the tumor. Each tumor was treated as an independent sample $(n=10)$. To measure the total uptake of TZB, the total area of green intensity in the tumor was divided by the total tumor area. To calculate the vascular density, an individual blood vessel image was segmented using the fuzzy c-means clustering method and converted to a binary image. The fraction of vessel area over the entire tumor area was determined to obtain the vascular density $(\%)$.

Because $\mathrm{Ab}$ penetrated into the tissues from both tumor surface and blood vessels, ${ }^{15}$ 20-line profiles were plotted from tumor surface and blood vessel (0-150 $\mu \mathrm{m})$ on 10 tumor samples. Each tumor was treated as an independent sample $(n=10)$. All line profiles from each group were analyzed using an in-house program written in MATLAB (MathWorks, Natick, Massachusetts, USA). The line profile intensity was averaged using the following equation:

$$
I\left(x_{i}\right)=\frac{1}{N_{i j}} \sum_{j=1}^{N_{j}} \sum_{n} \phi_{n i}\left(a_{j n}\right) \times f_{j}\left(a_{j n}\right)
$$

where $N_{j}=$ the number of samples (the number of line profiling),

$$
\begin{gathered}
a_{j n} \in \text { line profiling data }\left(\mathrm{j}^{\prime} \text { s sample }\right) \\
a_{j n} \in x_{j}=\text { line profiling data }\left(\mathrm{j}^{\prime} \text { s sample }\right)
\end{gathered}
$$

$$
\begin{gathered}
f_{j}(x)=\text { line profiling intensity data }\left(\mathrm{j}^{\prime} \text { s sample }\right) \\
\Delta x_{i}=\left[x_{i}-\frac{h}{2}+h \times(i-1), x_{i}+\frac{h}{2}+h \times(i-1)\right] \\
\text { for } \forall_{j} N_{i j}=n\left(\sum_{j}\left(x_{j} \cap \Delta_{x i}\right)\right)
\end{gathered}
$$

To identify the difference in Ab uptake, the area under the curve (AUC) was quantified. AUC was represented as mean \pm SD.

\section{Assessment of antitumor efficacy by combination therapy of RLX-expressing oncolytic AD and $\alpha \mathrm{PD}-1$}

Syrian golden hamsters (Japan SLC, Tokyo, Japan) were maintained in a laminar airflow cabinet under specific pathogen-free conditions. All facilities were approved by the Association and Accreditation of Laboratory Animal Care.

For the subcutaneous tumor model, tumors were implanted subcutaneously on the right flank of Syrian golden hamsters by inoculating $3 \times 10^{6}$ HaP-T1 cells suspended in $50 \mu \mathrm{L}$ of Hank's balanced salt solution (GIBCO-BRL). To assess the antitumor effect of various treatments, oAd/IL12/GM-RLX and $\alpha$ PD-1, PBS, $\alpha$ PD-1 $(10 \mathrm{mg} / \mathrm{kg})$, oAd/IL12/GM-RLX $\left(7 \times 10^{7}\right.$ or $\left.1 \times 10^{9} \mathrm{VP}\right)$, or oAd/IL12/GM-RLX $\left(7 \times 10^{7}\right.$ or $\left.1 \times 10^{9} \mathrm{VP}\right)$ plus $\alpha$ PD- 1 (10 $\mathrm{mg} / \mathrm{kg}$ ) were administered to the tumor-bearing hamsters. The first day of treatment was designated as day 0 . To assess the antitumor efficacy of each therapeutic in ICI refractory tumors, $\alpha$ PD- $1(10 \mathrm{mg} / \mathrm{kg})$ was administered at days 2,5 , and 8 to generate ICI refractory tumors, which were subsequently administered four times with oAd/IL12/GM-RLX $\left(1 \times 10^{9} \mathrm{VP}\right)$ at intervals of 2 days from day 9 . All treatments began when the average tumor volume was approximately $100 \mathrm{~mm}^{3}$. All treatments except $\alpha \mathrm{PD}-1$, which was administered via intraperitoneal injection, were administered intratumorally. Tumor growth was evaluated every day by taking measurements of the length (L) and width (W) of the tumor. Tumor volume was calculated using the following formula: volume $=0.523$ $\mathrm{L}(\mathrm{W})^{2}$.

For the orthotopic tumor model, HP-1 cancer cells $\left(4 \times 10^{5}\right)$ were injected directly into the tail of pancreas, and the establishment of the orthotopic pancreatic tumor model was confirmed by bioluminescence imaging as previously reported. ${ }^{16} \mathrm{HP}-1$ orthotopic pancreatic tumorbearing hamsters were administered $1 \times 10^{9} \mathrm{VP}$ of oAd/ IL12/GM-RLX into the abdomen twice (days 4 and 6), followed by a single injection into the tumor (day 8). $\alpha \mathrm{PD}-1$ was injected intraperitoneally at 3 day intervals three times (days 6, 9, and 12). The tumor volume was measured by luciferase signal at days 4, 9, and 13 after cancer cell injection.

\section{Histological and immunohistochemical analysis}

For histological analysis, tumor tissues were collected from hamsters at 10 days postinitial Ad injection, embedded in paraffin, and sectioned at $4 \mu \mathrm{m}$ thickness for $\mathrm{H} \& \mathrm{E}$ staining. For immunohistochemical staining, the $4 \mu$ m-thick tumor sections were blocked with $3 \%$ 
bovine serum albumin in Tris-buffered saline (Sigma, St. Louis, Missouri, USA) for 2 hours. The tumor sections were then incubated with a mouse anticollagen type I Ab (Cell Signaling Technology, USA) or a mouse antiproliferating cell nuclear antigen (PCNA) Ab (M0879; DAKO, Glostrup, Denmark) as primary Ab. After washing, the sections were incubated with goat anti-mouse IgG $\mathrm{Ab}$ $(\mathrm{H}+\mathrm{L})-\mathrm{HRP}$ (1031-05; Southern Biotech) as a secondary $\mathrm{Ab}$, and then counterstained with Meyer's hematoxylin (Sigma). A terminal deoxynucleotidyl transferase dUTP nick end labeling assay was performed as previously described. ${ }^{16}$ To identify lymphocyte infiltration into tumor tissues, tumor tissues were frozen in OCT compound (Sakura Finetec, Torrance, California, USA) and cut into $8 \mu \mathrm{m}$ sections. Tumor sections were fixed with chilled acetone for $10 \mathrm{~min}$ and blocked with Blocking Solution (DAKO). Sections were then incubated with primary Abs, mouse anti-rat CD4 monoclonal Ab (ebioscience, San Diego, California, USA) and mouse anti-rat CD8 monoclonal $\mathrm{Ab}$ (ebioscience) at $4^{\circ} \mathrm{C}$ overnight. After washing three times with PBS, samples were incubated with the secondary Ab, Alexa 647 goat anti-mouse Ab (Abcam, Cambridge, Massachusetts, USA) at room temperature for 2 hours. In the final step, the slides were washed with PBS, mounted with Prolong Gold antifade reagent with DAPI (Invitrogen), and then examined under a fluorescence microscope (IN Cell analyzer 2200).

\section{Preparation of radiolabeled $\mathbf{A b}$}

${ }^{64} \mathrm{Cu}$ is a radionuclide produced by a cyclotron with an intermediate half-life $\left(\mathrm{t}_{1 / 2}, 12.7 \mathrm{~h}\right)$ that decays by both $\beta+(655 \mathrm{keV}, 17.4 \%)$ and $\beta-(573 \mathrm{keV}, 39.0 \%)$ emission, making it suitable for both labeling Abs for positron emission tomography (PET) imaging of cancer. ${ }^{17}$ 1,4,7,10-Te traazacyclododecane-1,4,7,10-tetraacetic acid (DOTA)conjugated $\mathrm{Ab}$ (DOTA-Ab) was prepared by conjugating DOTA-NHS-ester (Macrocyclics, Dallas, Texas, USA) to $10 \mathrm{mg}$ of $\mathrm{Ab}$ with a 20-fold molar ratio in $0.1 \mathrm{M}$ sodium bicarbonate buffer, $\mathrm{pH}$ 8.5. The mixtures were incubated overnight with mild shaking at $4^{\circ} \mathrm{C}$, after which the conjugated Abs were purified from excess DOTA-NHS-ester with a PD-10 size exclusion column (GE Healthcare) using $1 \mathrm{mM}$ sodium acetate buffer, $\mathrm{pH} 6.5 .{ }^{64} \mathrm{CuCl}_{2}$ was produced by $50 \mathrm{MeV}$ cyclotron irradiation at the Korea Institute of Radiologic and Medical Sciences. ${ }^{18}$ DOTA-Ab $(3 \mathrm{mg})$ was incubated with ${ }^{64} \mathrm{Cu}(370 \mathrm{MBq})$ in $1 \mathrm{mM}$ sodium acetate buffer, $\mathrm{pH} 6.5$, for 1 hour at $37^{\circ} \mathrm{C}$. After incubation, ${ }^{64} \mathrm{Cu}$-conjugated DOTA-Ab was purified by use of a PD-10 size exclusion column that was eluted with $1 \mathrm{mM}$ sodium acetate buffer. The radiochemical purity of ${ }^{64} \mathrm{Cu}-\mathrm{Ab}$ was $\geq 98 \%$ by analysis with thin layer chromatography. The immunoreactivity of ${ }^{64} \mathrm{Cu}-\mathrm{TZB}$ or ${ }^{64} \mathrm{Cu}-\alpha \mathrm{PD}-1$ was determined to be $\geq 93 \%$ using a cell-binding assay.

\section{ImmunoPET imaging}

When the average tumor size in NCI-N87 tumor-bearing mice reached $200 \mathrm{~mm}^{3},{ }^{64} \mathrm{Cu}-\mathrm{TZB}(150 \mu \mathrm{g}, 14.8-18.5$ $\mathrm{MBq} / 200 \mu \mathrm{L}$ ) was administered on day 0 , whereas oAd/
$\operatorname{RLX}\left(2.5 \times 10^{7} \mathrm{VP}\right)$ was administered on days 0,2 , and 4 via intravenous injection. For imaging of ${ }^{64} \mathrm{Cu}-\alpha \mathrm{PD}-1, \mathrm{HaP}-\mathrm{T} 1$ tumor-bearing hamsters were intratumorally treated with oAd/IL12/GM-RLX $\left(7 \times 10^{7} \mathrm{VP}\right)$ on days 0 and 2 , whereas ${ }^{64} \mathrm{Cu}-\alpha \mathrm{PD}-1(400 \mu \mathrm{g}, 17.39-20.35 \mathrm{MBq} / 200 \mu \mathrm{L})$ was intraperitoneally injected on day 2. Before PET imaging with a Siemens Inveon PET scanner (Siemens Healthcare, Erlangen, Germany), mice or hamsters were anesthetized with $2.5 \%$ isoflurane. For the acquisition of anatomical images, CT imaging was acquired with the second bed position, full rotation, and 180 projections per bed position. The exposure time was $200 \mathrm{~ms}$ and the estimated scan time was $504 \mathrm{~s}$ for CT imaging. CT data were reconstructed using Feldkamp reconstruction with a SheppLogan filter. The effective pixel size of the reconstructed CT image was $109.69 \mu \mathrm{m} \times 109.69 \mu \mathrm{m}$. PET data were acquired for $15 \mathrm{~min}$ within an energy window of 350-650 $\mathrm{keV}$ at each time point after injection of ${ }^{64} \mathrm{Cu}-\mathrm{TZB}$ and ${ }^{64} \mathrm{Cu}-\alpha \mathrm{PD}-1$. The PET data were reconstructed with a $3 \mathrm{D}$ reprojection algorithm for regions of interest (ROI) analysis. The matrix size was $128 \times 128 \times 159$, and the voxel size was $0.776 \times 0.776 \times 0.796 \mathrm{~mm}^{3}$. PET and CT images were coregistered using Inveon Research Workplace V.2.0 (Siemens Healthcare). After coregistration of CT and PET data, the ROI was drawn on the CT image and copied to the PET data. \% ID /g was calculated by $\mathrm{C}_{\mathrm{T}} \cdot\left(\mathrm{V}_{\mathrm{T}} /\right.$ $\left.\mathrm{W}_{\mathrm{T}}\right) \cdot\left(1 / \mathrm{D}_{\mathrm{inj}}\right) \cdot 100 \%$, where $\mathrm{D}_{\mathrm{inj}}$ is the injected dose $(\mathrm{mCi})$; $\mathrm{C}_{\mathrm{T}}$ is the radioactivity in the tissue region $(\mathrm{mCi} / \mathrm{cc}) ; \mathrm{W}_{\mathrm{T}}$ is weight; and $\mathrm{V}_{\mathrm{T}}$ represents volume. Counts/second/voxel values were obtained from a PET ROI, and $\mathrm{mCi} / \mathrm{cc}$ tissue was calculated using a cylinder calibration factor. The density of tissue was assumed to be $\sim 1 \mathrm{cc}$ tissue/g of tissue.

\section{Autoradiography}

Immediately after PET/CT scanning, tumor tissues were isolated and frozen in OCT compound. After decaying for 48 hours, frozen tumors were sectioned at $20 \mu \mathrm{m}$ thickness using a cryostat microtome and exposed on an imaging plate for 24 hours. The plates were scanned with a BAS-5000 (Fujifilm, Tokyo, Japan). The signal intensities after tumor uptake of ${ }^{64} \mathrm{Cu}$-TZB or ${ }^{64} \mathrm{Cu}$-TZB plus oAd/ RLX were quantified as units of photostimulated luminescence per square millimetre (PSL $/ \mathrm{mm}^{2}$ ) using Multi Gage software V.3.0 (Fujifilm). For histological analysis, tumor tissues were stained by $\mathrm{H} \& \mathrm{E}$ and viewed under a light microscope (Carl Zeiss, Oberkochem, Germany).

\section{Biodistribution study}

After PET scanning at 60 hours after administration of ${ }^{64} \mathrm{Cu}-\alpha \mathrm{PD}-1$, hamsters ( $\mathrm{n}=3$, each group) were euthanized by $\mathrm{CO}_{2}$ gas asphyxiation and exsanguinated by cardiac pucture before dissection. The organs, tumor, and blood were collected and weighed. The decay-corrected radioactivity of the organs, tumor, and blood was measured as the total number of counts using a Wizard2 gammacounter (Perkin Elmer, Waltham, Massachusetts, USA). The activity data were represented as the percentage injected radioactivity dose per gram of tissue $(\% \mathrm{ID} / \mathrm{g})$. 


\section{Fluorescence-activated cell sorting (FACS) analysis}

HaP-T1 tumor-bearing hamsters were intratumorally injected with PBS, $\alpha$ PD-1 (10 mg/kg), oAd/IL12/ GM-RLX $\left(7 \times 10^{7} \mathrm{VP}\right)$, or oAd/IL12/GM-RLX $\left(7 \times 10^{7} \mathrm{VP}\right)$ plus $\alpha$ PD-1 $(10 \mathrm{mg} / \mathrm{kg})$. At 12 days after the initial treatment, lymphocytes were isolated from draining lymph node (DLN) or tumor tissue as previously reported. ${ }^{19}$ Before staining, cells were treated with saturating antiCD16/CD32 (Biolegend) in staining buffer (2\% FBS and $0.02 \%$ sodium azide in PBS). Then, cells were stained with hamster anti-mouse CD3e monoclonal Ab (BD Bioscience, San Jose, California, USA), mouse anti-rat CD4 monoclonal Ab (ebioscience), or mouse anti-rat CD8 monoclonal Ab (ebioscience) for the assessment of the CD4 or CD8 and interferon (IFN) $\gamma$ coexpressing T-cell population. After staining of surface markers (CD3, CD4, and CD8), cells were fixed and permeabilized with Cytofix/Cytoperm solution (BD pharMingen, San Jose, California, USA) and then stained with a rabbit antihamster IFN- $\gamma$ Ab (Abclon, Seoul, Korea). Samples were analyzed using a FACScan flow cytometer with CellQuest software (Beckton-Dickinson) as previously reported. ${ }^{19}$

\section{IFN- $\gamma$ ELISA}

Lymphocytes were harvested from PBS-treated, $\alpha$ PD-1treated, oAd/IL12/GM-RLX-treated or oAd/IL12/ GM-RLX plus $\alpha$ PD-1-treated hamsters and minced into single-cell suspensions in PBS containing 2\% FBS. The lymphocytes were then cocultured with irradiated HaP-T1 cells for 3 days in the presence of recombinant human IL-2 (Peprotech, Rocky Hill, New Jersey, USA). The supernatants were collected, and an IFN- $\gamma$ ELISA was carried out according to the manufacturer's protocol (Cusabio Biotech, Wuhan, China).

\section{Statistical analysis}

All statistical analyses were performed by two-tailed Student t-test or one-way analysis of variance (SPSS 13.0 software; SPSS, Chicago, Illinois, USA). Data are expressed as mean $\pm \mathrm{SD}$. $\mathrm{P}$ values less than 0.05 were assumed to denote statistical significance.

\section{RESULTS}

\section{Characterization of $\mathrm{OAd} / \mathrm{RLX}$}

Because dense and abnormal ECM functions as a physical barrier against drug dispersion, an oncolytic Ad expressing RLX (oAd/RLX, online supplementary figure S1A) was constructed to degrade tumor ECM. oAd/RLX expresses RLX in a dose-dependent manner (online supplementary figure S1B), decreases the accumulation of ECM in tumor tissues, and lowers the expression level of collagen by $80 \%$ compared with PBS-treated group (online supplementary figure $\mathrm{S} 1 \mathrm{C}-\mathrm{E}, * * * \mathrm{p}<0.001$ ).

To assess whether systemically administered oAd/RLX causes degradation of ECM in other organs, articular cartilage was isolated from oAd/RLX-treated and PBStreated mice, and the proteoglycan matrix was stained with Safranin-O (online supplementary figure S2). oAd/ RLX $\left(2.5 \times 10^{7} \mathrm{VP}\right.$, three times) had no adverse side effect on the proteoglycan matrix of fibrocartilage in the knee joint.

\section{Enhanced penetration of trastuzumab following oAd/RLX treatment}

determine whether oAd/RLX enhances the intratumoral penetration and accumulation of $\mathrm{Ab}$, tumor tissues from mice treated with Alexa 488-conjugated TZB (488-TZB) and/or oAd/RLX were isolated at 5 days after injection, and the localization of 488-TZB was observed. As shown in figure $1 \mathrm{~A}$, the quantity of 488-TZB was significantly increased in the oAd/RLX plus 488-TZB-treated tumors as compared with the 488-TZB-treated group. We further investigated 488-TZB accumulation in whole tumor tissues by analyzing fluorescence images. The accumulation of 488-TZB per tissue area was increased 2.9-fold in the 488-TZB-treated group compared with the oAd/RLX plus 488-TZB-treated group (figure 1B, ***p $<0.001$ ). We next determined if the increased $\mathrm{Ab}$ accumulation was associated with a change in blood vessel density in tumor tissues. As shown in figure 1C, the functional blood vessel density was not significantly changed after oAd/RLX treatment. These results suggest that oAd/RLX enhances the accumulation and penetration of therapeutic Abs in tumor tissue by inducing ECM remodeling in the tumor microenvironment, without changing the blood vessel density.

\section{Enhanced distribution of trastuzumab from the tumor edge and blood vessels induced by $0 A d / R L X$}

To determine how oAd/RLX affects the tumor infiltration of 488-TZB, we plotted line profiles indicating the distribution of 488-TZB relative to the tumor edge on various layers of the fluorescence images (figure 2A). The infiltration depth of 488-TZB was greatest in the oAd/RLX plus 488-TZB group; the largest fraction of 488-TZB accumulation occurred at $61.2 \mu \mathrm{m}$ from the tumor surface and the accumulation decreased in a distance-dependent manner (figure 2B). Importantly, improved penetration of therapeutic Abs led to enhanced $\mathrm{Ab}$ accumulation in tumor tissues in the oAd/RLX plus 488-TZB-treated group, resulting in 2.8-fold greater accumulation than in tumor tissues treated with 488-TZB alone (figure 2C, $* * * \mathrm{p}<0.001)$.

The intensity profile of 488-TZB was analyzed relative to blood vessels within tumor tissue (online supplementary figure S3A). 488-TZB penetrated deeper into blood vessels when used in conjunction with oAd/RLX than did 488-TZB alone, resulting in 3.2-fold greater 488-TZB accumulation in the blood vessel (online supplementary figure S3B,C, $* * * p<0.001)$. These findings suggest that the degradation of tumor stromal components by oAd/ RLX could improve tissue penetration of therapeutic Abs, resulting in enhanced drug distribution further away from the tumor edge and blood vessels. 
A

TZB

DAPI

Vessel
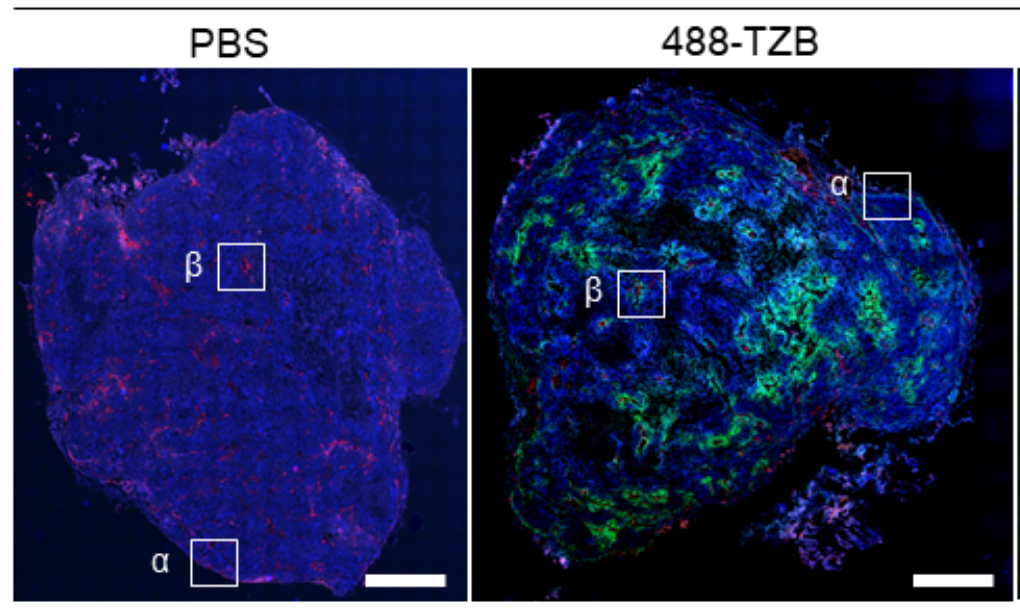

oAd/RLX+488-TZB

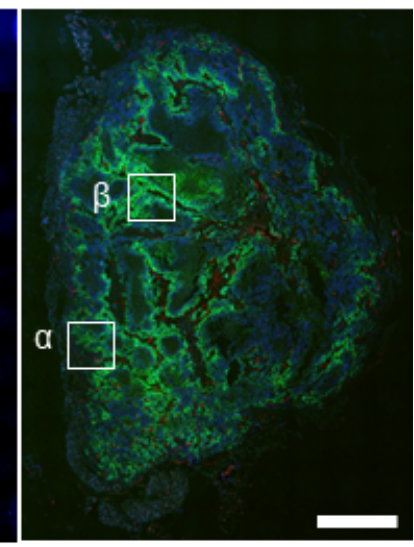

B
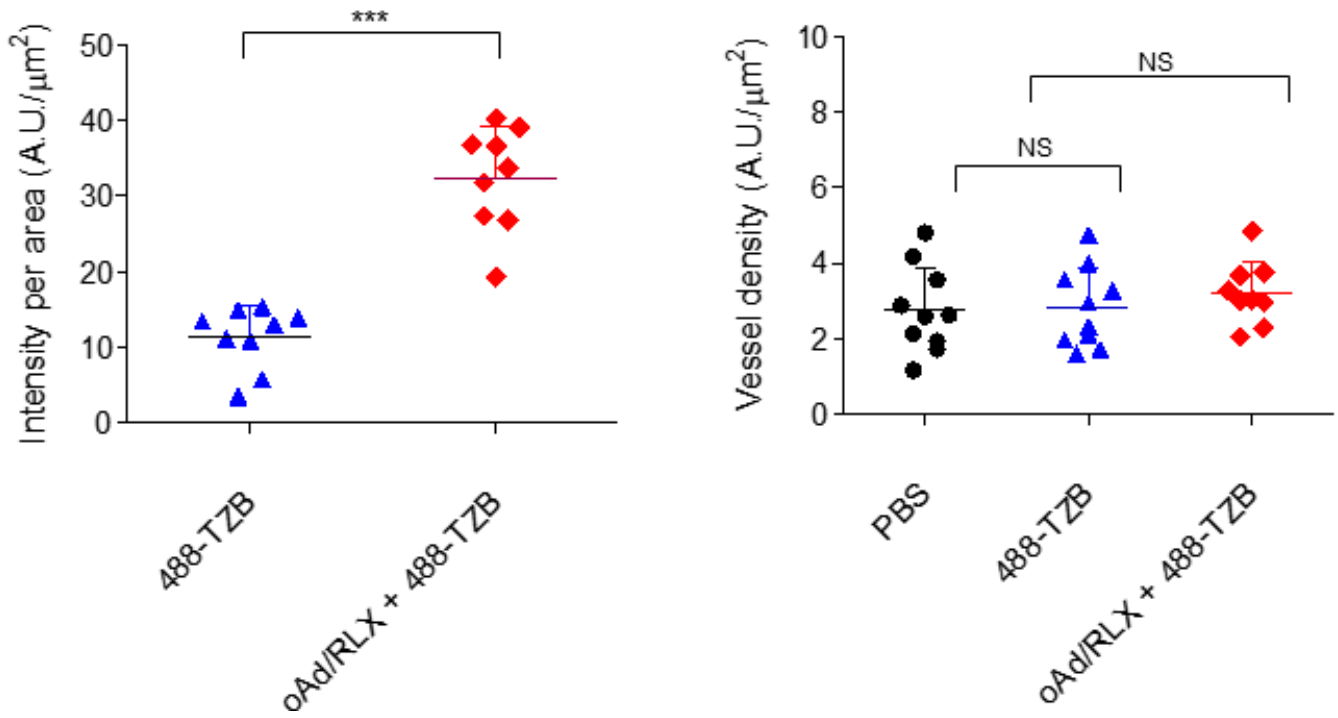

Figure 1 Distribution of TZB in whole tumor tissue. (A) Fluorescence image of 488-TZB (green) in NCl-N87 xenografted tumors. Tumors were isolated at 5 days after injection of 488-TZB or oAd/RLX plus 488-TZB into NCI-N87-xenograft mice. Blood vessels (red) and nuclei (blue) are shown for each group. The white boxes correspond to the regions displayed in figure 2A and online supplementary figure S3A. The scale bar represents $1 \mathrm{~mm}$. (B) The total accumulation of 488-TZB per tumor tissue area. The total accumulation of 488-TZB in the whole tumor region was measured from whole tissue fluorescence images (four images per mouse, mouse $n=10$ ). 488 -TZB versus $\circ A d / R L X+488$ TZB, ${ }^{\star \star \star} p<0.001$, statistical analyses were performed by two-tailed Student t-test. (C) The density of rhodamine-lectin-positive functional blood vessels. The vascular density was measured using the red signals from rhodamine-lectin-stained functional blood vessel images (four images per mouse, mouse $n=10$ ). To calculate the vascular density, an individual blood vessel image was segmented using the fuzzy C-means clustering method and converted to a binary image. The fraction of vessel area over the entire tumor area was determined to obtain the vascular density (\%). Quantitative data are presented as mean optical density $\pm S D(n=10)$. TZB, trastuzumab; DAPI, 4,6-diamidino-2-phenyindole; PBS, phosphate-buffered saline; 488-TZB, Alexa 488-conjugated trastuzumab; NS, not significant; oAd/RLX, relaxin-expressing oncolytic adenovirus.

\section{Increased accumulation of trastuzumab in tumor tissues induced by $0 \mathrm{Ad} / \mathrm{RLX}$}

To evaluate the penetration of $\mathrm{Ab}$ into tumors, PET imaging of ${ }^{64} \mathrm{Cu}$-DOTA-TZB $\left({ }^{64} \mathrm{Cu}\right.$-TZB $)$ and/or oAd RLX injected mice was conducted. As shown in figure 3A, higher uptake of ${ }^{64} \mathrm{Cu}$-TZB was observed in tumors treated with a combination of oAd/RLX plus ${ }^{64} \mathrm{Cu}-\mathrm{TZB}$ than in those treated with ${ }^{64} \mathrm{Cu}-\mathrm{TZB}$ at all time $(* * * \mathrm{p}<0.001)$. Autoradiography showed a greater tumor uptake of ${ }^{64} \mathrm{Cu}-$ TZB in the oAd/RLX plus ${ }^{64} \mathrm{Cu}$-TZB-treated group than in the ${ }^{64} \mathrm{Cu}$-TZB-treated group (figure $3 \mathrm{~B},{ }^{*} \mathrm{p}<0.01$ ). The enhancement in ${ }^{64} \mathrm{Cu}$-TZB accumulation was tumorspecific because radioisotope accumulation was similar in normal tissues in the ${ }^{64} \mathrm{Cu}-\mathrm{TZB}$-treated group and oAd/ 
A

TZB

DAPI

Vessel
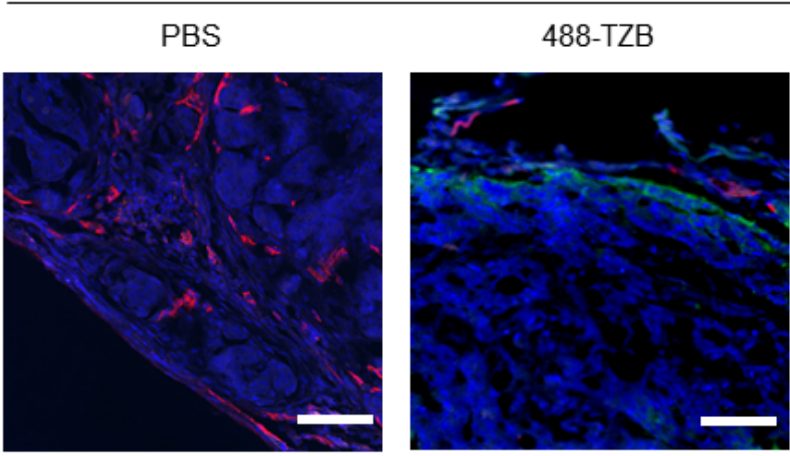

$\mathrm{oAd} / \mathrm{RLX}+488-\mathrm{TZB}$

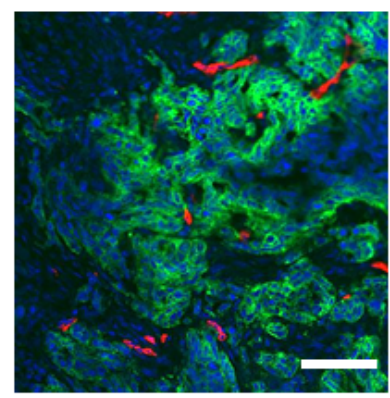

B
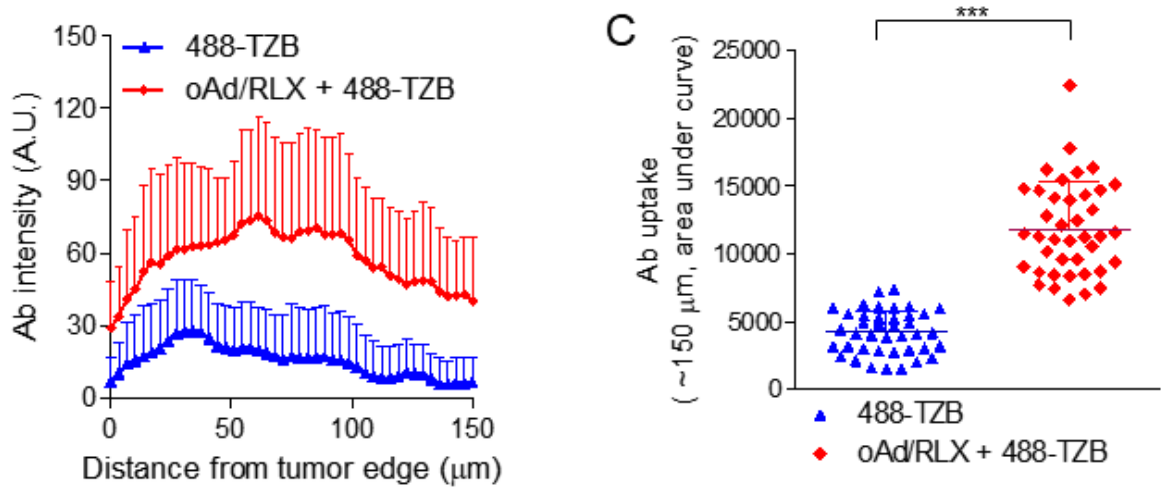

Figure 2 In vivo accumulation and penetration of 488-TZB relative to the tumor edge and functional blood vessels. (A) Fuorescence images magnified from the white boxes labeled $\alpha$ in figure 1A. 488-TZB (green), rhodamine-lectin-positive functional blood vessels (red), and DAPI-stained nuclei (blue) are shown for each group. The scale bar represents $100 \mu \mathrm{m}$. (B) Quantitative analysis of 488-TZB intensity in tumor tissue (0-150 $\mu \mathrm{m}$ from the tumor edge). (C) Uptake of 488-TZB quantified by area under curve analysis $(0-150 \mu \mathrm{m}$ from the tumor edge). Quantitative data are presented as mean optical density $\pm S D(n=10)$; 488-TZB versus $\circ A d / R L X+488 \mathrm{TZB},{ }^{* \star *} \mathrm{p}<0.001$. Statistical analyses were performed by two-tailed Student $\mathrm{t}$-test. Ab, antibody; TZB, trastuzumab; DAPI, 4,6-diamidino-2-phenyindole; PBS, phosphate-buffered saline; 488-TZB, Alexa 488-conjugated trastuzumab; oAd/RLX, relaxin-expressing oncolytic adenovirus.

RLX plus ${ }^{64} \mathrm{Cu}$-TZB-treated groups (online supplementary table S1). Together, these results suggest that ${ }^{64} \mathrm{Cu}-$ TZB can be used safely in combination with oAd/RLX with minimal off-target toxicity.

\section{Potent therapeutic efficacy of the IL-12, GM-CSF, and RLX- coexpressing oncolytic Ad in combination with $\alpha \mathrm{PD}-1$ in a Syrian hamster tumor model}

We have designed and constructed an oncolytic Ad coexpressing antitumor cytokines (IL-12 and GM-CSF) and RLX (oAd/IL12/GM-RLX; online supplementary figure S4A), ultimately aiming to restore antitumor immune functions in the tumor milieu and enhance the intratumoral penetration of the oncolytic Ad, ICIs, and immune cells through ECM degradation. The expression of three therapeutic genes by oAd/IL12/GM-RLX increased in a dose-dependent manner (online supplementary figure S4B). These results demonstrate that single vector arming three functional therapeutic genes can express all therapeutic genes effectively in cancer cells. Next, we analyzed whether the combined expression of three therapeutic genes translate to more potent antitumor effect than oncolytic Ad expressing either RLX alone $(\mathrm{oAd} / \mathrm{RLX})^{13} 20$ or coexpressing IL-12 and GM-CSF (oAd/IL12/GM) ${ }^{21}$ in a Syrian hamster syngeneic pancreatic tumor model. As shown in online supplementary figure S9, all therapeutic gene expressing oAds (oAd/RLX, oAd/IL12/GM, and oAd/ IL12/GM-RLX) elicited more robust tumor growth inhibition than control oAd. Antitumor cytokine expressing oAds (oAd/IL12/GM and oAd/IL12/GM-RLX) elicited more potent antitumor effect than oAd or oAd/RLX, in line with reports showing that immune boosting genes are integral to maximizing antitumor effect of oncolytic viruses in field of immuno-oncology. Importantly, oAd/ IL12/GM-RLX had a more potent antitumor effect than $\mathrm{oAd} / \mathrm{IL} 12 / \mathrm{GM}(* * * \mathrm{p}<0.001)$, showing that addition of RLX improved overall potency of oAd (online supplementary figure S9A). In detail, oAd/IL12/GM-RLX induced higher intratumoral expression of E1A in tumor tissues than oAd/IL12/GM $(* * * p<0.001)$, while the capacity of the virus to induce degradation of ECM components in tumor tissues were retained at similar level to oAd/RLX (online supplementary figure S9B). Further, oAd/IL12/GM-RLX induced a higher level of 

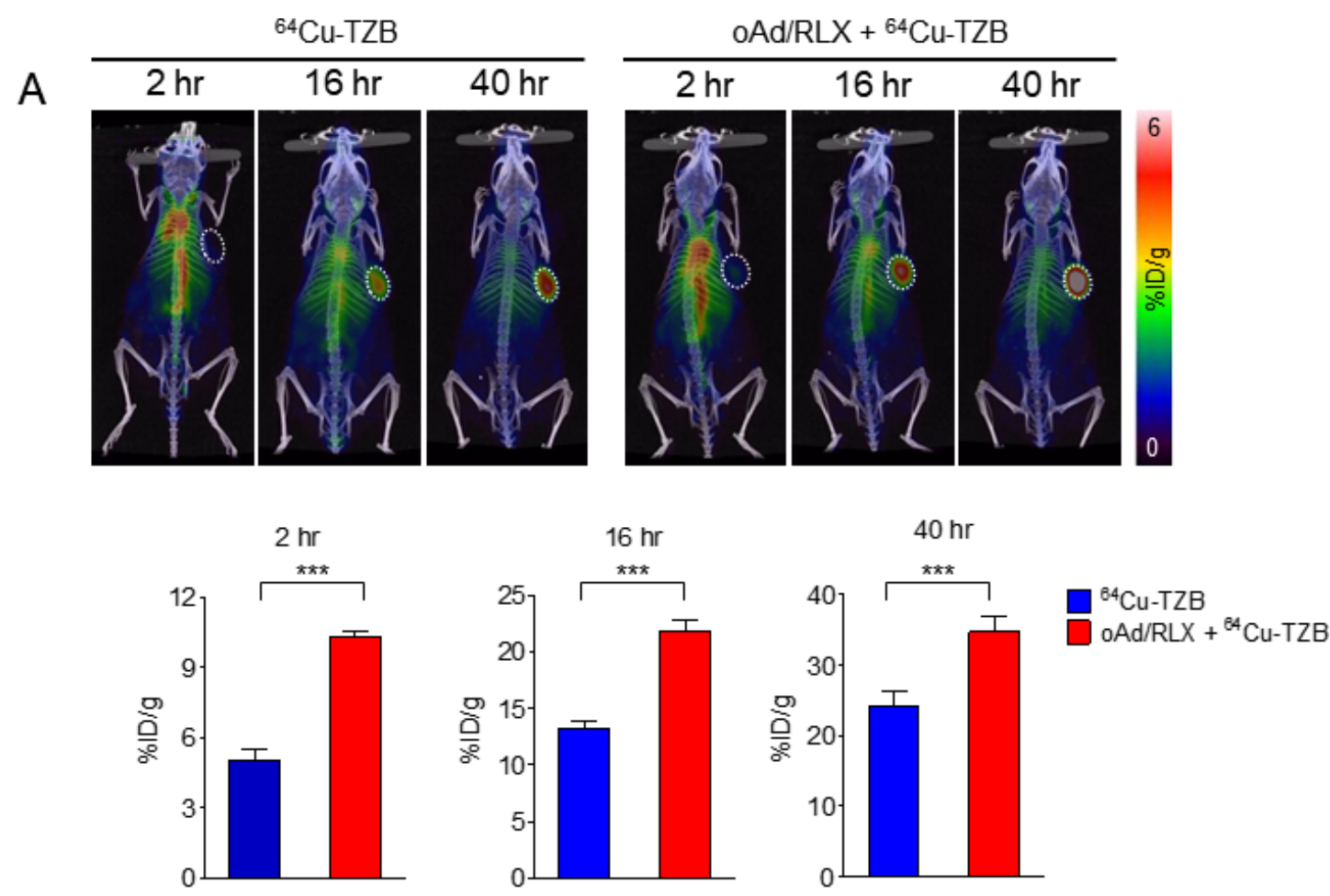

B
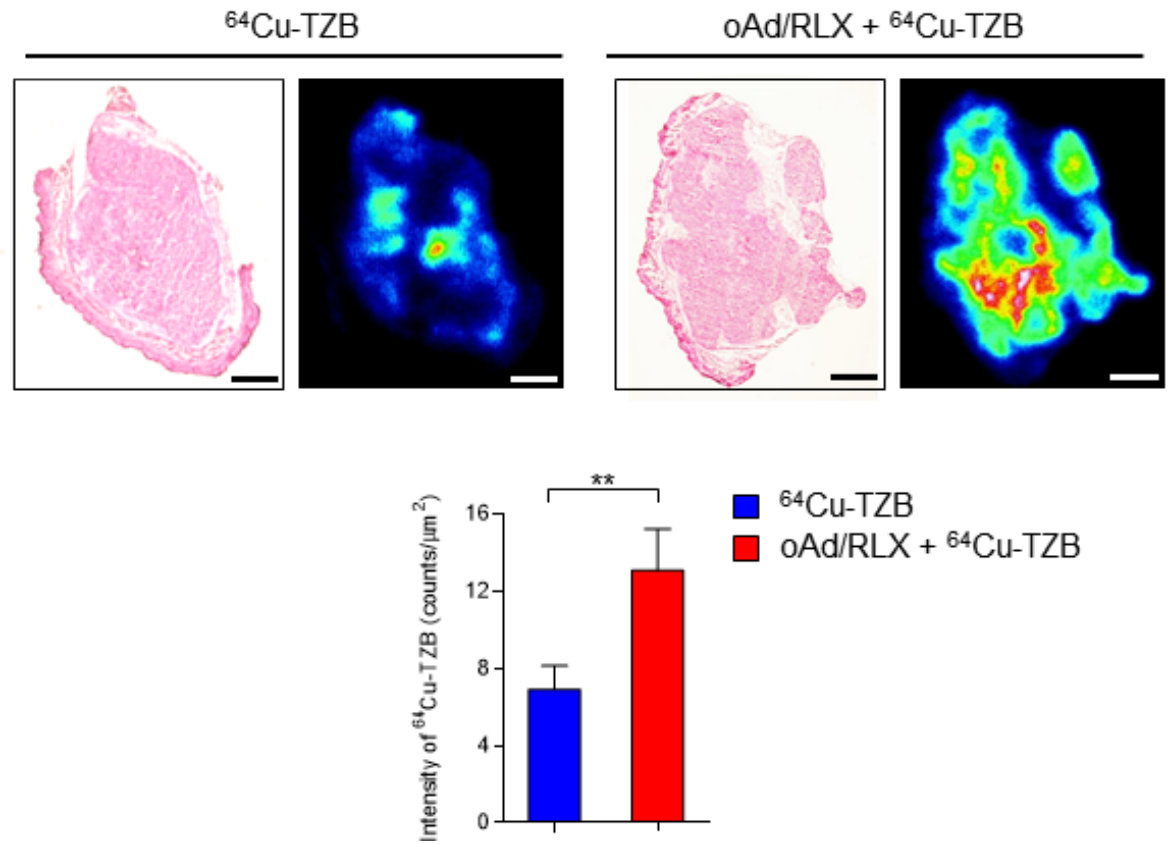

Figure 3 ImmunoPET images. (A) Representative PET/CT images of NCI-N87 tumor-bearing mice at 2, 16, and 40 hours after injection of ${ }^{64} \mathrm{Cu}$-TZB alone (left) or oAd/RLX plus ${ }^{64} \mathrm{Cu}-\mathrm{TZB}$ (right). White dotted circles indicate the tumor regions. Quantitative data are presented as mean $\pm S D(n=5) ;{ }^{64} \mathrm{Cu}$-TZB versus oAd/RLX plus ${ }^{64} \mathrm{Cu}-\mathrm{TZB}$, ${ }^{\star * *} \mathrm{p}<0.001$. Statistical analyses were performed by by two-tailed Student t-test. (B) Quantification of the intratumoral distribution of ${ }^{64} \mathrm{Cu}-\mathrm{TZB}$ in $\mathrm{NCl}-\mathrm{N} 87$ tumors by H\&E staining and autoradiography. After acquisition of PET images, tumors were isolated from mice. Tumor sections were exposed to an image plate for scanning. The scale bar represents $1 \mathrm{~mm}$. Quantitative data are presented as mean optical density $\pm S D(n=3) ;{ }^{64} \mathrm{Cu}-\mathrm{TZB}$ versus oAd/RLX plus ${ }^{64} \mathrm{Cu}-\mathrm{TZB},{ }^{\star *} \mathrm{p}<0.01$. Statistical analyses were performed by two-tailed Student t-test. TZB, trastuzumab; oAd/RLX, relaxin-expressing oncolytic adenovirus; PET, positron emission tomography. 
intratumoral T-cell infiltration $\left(\mathrm{CD} 4^{+}\right.$and $\mathrm{CD} 8^{+} \mathrm{T}$ cells $)$ and IFN- $\gamma$ expression compared with oAd/IL12/GM (online supplementary figure S9C and S9D, *** $\mathrm{p}<0.001$ or $* \mathrm{p}<0.05)$. Together, these results show that addition of RLX improves ECM degradation, intratumoral viral dispersion, and induction of antitumor immune response.

To evaluate the combined antitumor efficacy of oAd/ IL12/GM-RLX and $\alpha$ PD-1, Syrian hamsters were subcutaneously injected with HaP-T1 pancreatic cancer cells. The tumor-bearing hamsters were treated with $\alpha \mathrm{PD}-1$, oAd/IL12/GM-RLX, or oAd/IL12/GM-RLX plus $\alpha \mathrm{PD}-1$, along with PBS as a negative control. As shown in figure $4 \mathrm{~A}$, tumors treated with PBS exhibited robust growth with the tumor volume reaching $2913 \pm 95 \mathrm{~mm}^{3}$ at 23 days following the initial treatment. The $\alpha$ PD-1-treated group showed modest tumor growth inhibition, with a $33 \%$ reduction in the average tumor volume in comparison with the PBS control group $\left({ }^{*} \mathrm{p}<0.05\right)$. Importantly, both oAd/IL12/GM-RLX and oAd/IL12/GM-RLX plus $\alpha \mathrm{PD}-1$ treatments elicited significantly more potent antitumor effects than either PBS or $\alpha$ PD-1 monotherapy $(* * * \mathrm{p}<0.001)$, resulting in complete tumor regression in all six hamsters tested. To assess the establishment of adaptive immunity against the tumor re-establishment following each treatment, hamsters with complete tumor regression were rechallenged at 61 days postinoculation of the primary tumor. All of the rechallenged tumors regressed in both the oAd/IL12/GM-RLX monotherapy and $\alpha$ PD- 1 combination treatment groups (figure $4 \mathrm{~B}$ ), demonstrating that oAd/IL12/GM-RLX was sufficient to establish durable adaptive immunity against the tumor re-establishment.

Because it was difficult to determine whether combination therapy was more effective than oncolytic Ad monotherapy when using oAd dose of $1 \times 10^{9} \mathrm{VP}$ as in figure $4 \mathrm{~A}$, the antitumor efficacy of oAd/IL12/GM-RLX and oAd/ IL12/GM-RLX plus $\alpha$ PD-1 treatment was re-evaluated again at a 14.3 -fold lower viral dose $\left(7 \times 10^{7} \mathrm{VP}\right)$. As shown in figure 4C, oAd/IL12/GM-RLX-treated tumors continued to grow and reached $1982 \pm 126 \mathrm{~mm}^{3}$ by day 30 post-treatment. In marked contrast, oAd/IL12/GM-RLX plus $\alpha \mathrm{PD}-1$ treatment suppressed tumor growth, resulting in $79 \%$ tumor growth inhibition compared with the monotherapy group $(* * * \mathrm{p}<0.001)$. Importantly, $50 \%$ of the hamsters in the oAd/IL12/GM-RLX plus $\alpha$ PD-1 group showed complete tumor regression, whereas none of the six tumors in the monotherapy group showed complete regression. Biodistribution profiling of Ad revealed that Ad was distributed in a similar manner in oAd/IL12/ GM-RLX-treated and oAd/IL12/GM-RLX plus $\alpha$ PD-1treated mice, with the highest level being detected in tumor tissues (online supplementary figure S7). Likely due to the low level of Ad accumulation in normal organs following administration of either oAd/IL12/GM-RLX or oAd/IL12/GM-RLX plus $\alpha$ PD-1, no liver and kidney toxicities were observed, as various serum markers fell within the conventional range reported by others (online supplementary figure S8). ${ }^{22-24}$

An orthotopic pancreatic tumor model in Syrian hamster has been reported to closely recapitulate morphological and biological aspects of pancreatic cancer in humans. ${ }^{25}$ Thus, the antitumor effect of each treatment was further assessed in an orthotopic HP-1 pancreatic tumor model in Syrian hamsters. As shown in figure 4E, $\alpha \mathrm{PD}-1$ monotherapy failed to efficiently suppress orthotopic tumor growth. Orthotopic pancreatic tumors were highly aggressive and metastatic, as both PBS-treated and $\alpha$ PD-1treated groups showed extensive metastasis to different sites and mortality in the subpopulation of hamsters by 11 days after the initial treatment (online supplementary figure S6A). Additionally, significant ascites accumulation, which is a prognostic marker for pancreatic cancer progression and mortality in clinic, ${ }^{26}$ was observed in PBStreated or $\alpha \mathrm{PD}$-1-treated hamsters. In sharp contrast, both oAd/IL12/GM-RLX and oAd/IL12/GM-RLX plus $\alpha$ PD-1 induced significantly more potent primary and metastatic tumor growth inhibition, as well as complete prevention of ascites accumulation, in respect to $\alpha \mathrm{PD}-1$ monotherapy group (online supplementary figure $\mathrm{S} 4 \mathrm{E}$ and $\mathrm{S} 6$ ). This is in line with previous report demonstrating that an oAdmediated RLX expression has been shown to inhibit tumor metastasis, ${ }^{13}$ showing that localized overexpression of RLX by oAd exerts an antimetastatic effect. Collectively, these results demonstrate that oAd/IL12/GM-RLX in combination with $\alpha \mathrm{PD}-1$ can induce potent antitumor efficacy against pancreatic tumors with no observable side effects.

\section{Histological and immunohistochemical analyses}

To further investigate the therapeutic effect of combination treatment, tumor tissues harvested from hamsters were assessed histologically and immunohistologically. As shown in figure $4 \mathrm{D}$, necrotic regions were rarely detectable in tumors in the PBS-treated and $\alpha \mathrm{PD}-1$-treated groups, whereas oAd/IL12/GM-RLX plus $\alpha$ PD-1-treated tumors were mostly necrotic as revealed by $\mathrm{H} \& \mathrm{E}$ staining. Moreover, oAd/IL12/GM-RLX plus $\alpha$ PD-1-cotreated tumors showed a much lower density of proliferating tumor cells (PCNA-positive) than did tumors treated with monotherapy, suggesting that the combination of oncolytic Ad with $\alpha$ PD-1 inhibits tumor cell proliferation more effectively than monotherapy can. Furthermore, oAd/ IL12/GM-RLX plus $\alpha$ PD-1-treated tumors contained a larger area with an apoptotic tumor cell population than did tumors treated with monotherapy, indicating that attenuated tumor cell proliferation correlated with an increased induction of apoptosis.

\section{Increased tumor penetration of $\alpha \mathrm{PD}-1$ after treatment with OAd/IL12/GM-RLX}

The distribution of a therapeutic Ab, TZB, in tumor tissue was enhanced by oncolytic Ad-mediated intratumoral expression of RLX (figures 1-3). On the basis of these results, we hypothesized that a combination of oAd/ 

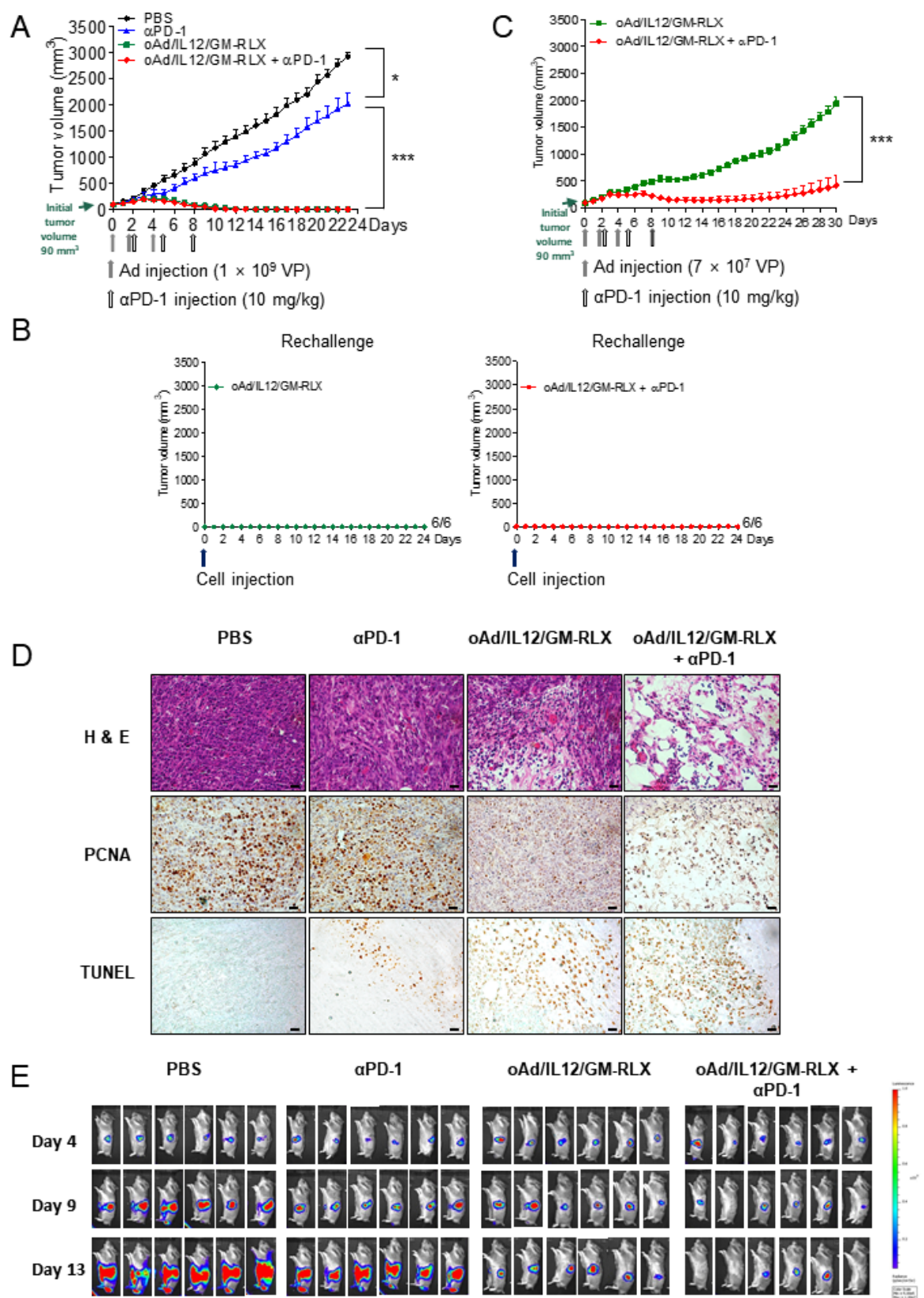

Figure 4 Antitumor efficacy of oAd/IL12/GM-RLX and $\alpha \mathrm{PD}-1$ combination therapy and histological and histochemical analyses. Effect of oAd/IL12/GM-RLX and $\alpha \mathrm{PD}-1$ combination therapy on tumor volume over time. Syrian hamsters were subcutaneously injected with HaP-T1 cells to establish pancreatic tumors. When the average tumor volume reached 90-100 $\mathrm{mm}^{3}$, (A) the tumors were injected with $1 \times 10^{9} \mathrm{VP}$ of oAd/IL12/GM-RLX (days 0,2 , and 4; gray arrows) and/or $10 \mathrm{mg} / \mathrm{kg}$ of $\alpha P D-$ 1 (days 2, 5, and 8; empty arrows). The tumor volume was measured every day until the end of the study. Data are presented as mean $\pm S D(n=6)$; PBS versus $\alpha P D-1,{ }^{*} p<0.05 ; \alpha P D-1$ versus $\circ A d / / L 12 / G M-R L X$ or $\circ A d / / L 12 / G M-R L X+\alpha P D-1,{ }^{* \star *} p<0.001$. Statistical analyses were performed by one-way analysis of variance. (B) Rechallenged tumor growth. Hamsters with complete regression $(A)$ were rechallenged at 61 days postinoculation of the primary tumor. The PBS-treated and $\alpha$ PD-1-treated groups were excluded from the rechallenge experiment, as these hamsters had been euthanized due to tumor burden exceeding the ethical guidelines of our institution at the time of the rechallenge experiment. (C) The tumors were injected with $7 \times 10^{7}$ VP of $\mathrm{oAd} / \mathrm{IL} 12 / \mathrm{GM}-\mathrm{RLX}$ (days 0 , 2, and 4; gray arrows) with or without $10 \mathrm{mg} / \mathrm{kg}$ of $\alpha \mathrm{PD}-1$ (days 2, 5, and 8; empty arrows). The tumor volume was measured every day until the end of the study. Data are presented as mean $\pm S D(n=6)$; oAd/IL12/GM-RLX

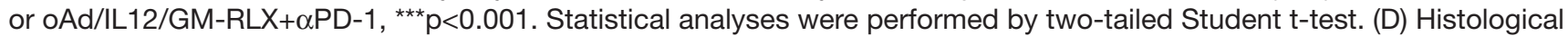
and immunohistological analysis. Tumor tissues were collected on day 10 after the first virus injection and stained with H\&E, an anti-PCNA antibody, or TUNEL. Original magnification: $\times 400$. The scale bar represents $2 \mu \mathrm{m}$. (E) HP-1 orthotopic pancreatic tumor-bearing hamsters were administered $1 \times 10^{9} \mathrm{VP}$ of oAd/IL12/GM-RLX into the abdomen twice (days 4 and 6), followed by a single injection into the tumor (day 8). $\alpha$ PD-1 was injected intraperitoneally at 3-day intervals three times (days 6, 9, and 12). The tumor volume was measured by luciferase signal at days 4, 9, and 13 after cancer cell injection. PBS, phosphate-buffered saline; $\alpha \mathrm{PD}-1$, anti-programmed cell death protein 1; Ad, adenovirus; PCNA, proliferating cell nuclear antigen; TUNEL, terminal deoxynucleotidyl transferase dUTP nick end labeling; VP, viral particle. 
IL12/GM-RLX and $\alpha$ PD-1 might elicit synergistic antitumor effects because RLX would facilitate the intratumoral penetration of $\alpha \mathrm{PD}-1$. To investigate whether oAd/ IL12/GM-RLX increased the intratumoral penetration of $\alpha \mathrm{PD}-1$ into desmoplastic pancreatic tumor tissues, oAd/ IL12/GM-RLX alone, Alexa 488-conjugated $\alpha$ PD-1 alone (488- $\alpha$ PD-1), or a combination of these treatments was administered. $\alpha$ PD-1As shown in figure 5A,B, the amount of $\alpha$ PD-1 penetration in tumor tissue increased 2.7-fold in the combination therapy group compared with $\alpha \mathrm{PD}-1$ monotherapy group at 7 days of postinitial treatment (***p<0.001), demonstrating that oAd/IL12/GM-RLX enhances the penetration of $\alpha \mathrm{PD}-1$ into tumor.

To further evaluate the tumor uptake of $\alpha \mathrm{PD}-1$ and its whole-body distribution, we acquired immunoPET images of HaP-T1 tumor bearing hamsters injected with ${ }^{64} \mathrm{Cu}$-conjugated $\alpha \mathrm{PD}-1\left({ }^{64} \mathrm{Cu}-\alpha \mathrm{PD}-1\right)$ with/without oAd/ IL12/GM-RLX in the same regimen as described earlier (figure 5C). The intratumoral uptake of ${ }^{64} \mathrm{Cu}-\alpha \mathrm{PD}-1$ in the combination therapy group was higher than that in the ${ }^{64} \mathrm{Cu}-\alpha \mathrm{PD}-1$ monotherapy group at all time points. The biodistribution of ${ }^{64} \mathrm{Cu}-\alpha \mathrm{PD}-1$ was calculated as $\% \mathrm{ID} / \mathrm{g}$ in various organs and tumor tissues at 60 hours of postinjection (figure 5D). The uptake of ${ }^{64} \mathrm{Cu}-\alpha \mathrm{PD}-1$ to tumor tissues or spleen was significantly higher in the combination therapy group than in the monotherapy group (1.56fold or 0.84 -fold higher in respective tissues, $* \mathrm{p}<0.05)$. In addition, the volume of DLN, a $\mathrm{T}$ cell-enriched site, increased by 48-fold following oAd/IL12/GM-RLX treatment (online supplementary figure S5). Taken together, these results suggest that a combination of oAd/IL12/ GM-RLX and $\alpha$ PD-1 enhances T-cell expansion and activation as well as augments $\alpha \mathrm{PD}-1$ colocalization in T cellenriched tissues to promote robust antitumor immunity.

\section{Increased cytotoxic T-cell activation and infiltration into tumor induced by coexpression of IL-12, GM-CSF, and RLX}

To explore whether increased penetration of $\alpha \mathrm{PD}-1$ into tumor induces the infiltration and activation of $\mathrm{T}$ cells, the accumulation of IFN- $\gamma$-expressing $\mathrm{CD} 4^{+}$ or $\mathrm{CD}^{+} \mathrm{T}$ cells was assessed in the DLN, which functions as an initial site of expansion for cytotoxic T cells. The number of IFN- $\gamma$-expressing $\mathrm{CD}^{+} \mathrm{T}$ cells in DLN of oAd/IL12/GM-RLX plus $\alpha$ PD-1 and oAd/IL12/ GM-RLX groups were similarly high, while the number of IFN- $\gamma$-expressing $\mathrm{CD} 4^{+} \mathrm{T}$ cells was highest in the DLN of oAd/IL12/GM-RLX plus $\alpha$ PD-1 group (figure 6A, $* * * \mathrm{p}<0.001$ ), suggesting a greater number of activated antitumor $\mathrm{T}$ cells are present in the lymphoid organ following combination therapy. Even if large quantities of activated $\mathrm{T}$ cells are present in various immune organs such as the DLN, antitumor immune responses may still be inadequate for clinical benefit because of inefficient intratumoral infiltration of these activated $\mathrm{T}$ cells. ${ }^{27}$ Thus, the intratumoral infiltration of activated $\mathrm{T}$ cells $\left(\mathrm{CD} 4^{+} \mathrm{IFN}-\gamma^{+} \mathrm{T}\right.$ cell and $\mathrm{CD} 8^{+} \mathrm{IFN}-\gamma^{+} \mathrm{T}$ cell subsets of the tumor-infiltrating lymphocytes (TILs)) was evaluated by flow cytometry. As shown in figure $6 \mathrm{~B}$, both $\mathrm{CD}^{+} \mathrm{IFN}-\gamma^{+}$
$\mathrm{T}$ cells and $\mathrm{CD} 8^{+} \mathrm{IFN}-\gamma^{+} \mathrm{T}$ cells accumulated at a higher frequency in combination therapy-treated tumors than in the $\alpha$ PD-1 or oAd/IL12/GM-RLX monotherapy groups $(* * * \mathrm{p}<0.001)$, suggesting that concomitant administration of immunostimulatory oncolytic Ad and $\alpha \mathrm{PD}-1$ can lead to enhanced $\mathrm{T}$ cell-mediated antitumor immune responses. These results were also confirmed by immunofluorescence staining of tumor tissues treated with PBS, $\alpha \mathrm{PD}-1$, or oAd/IL12/GM-RLX plus $\alpha \mathrm{PD}-1$. As shown in figure 6C,D, both $\mathrm{CD} 8^{+} \mathrm{IFN}-\gamma^{+}$and $\mathrm{CD}^{+}$perforin ${ }^{+} \mathrm{T}$ cells were more frequently detected in oAd/IL12/GM-RLX plus $\alpha$ PD-1-treated tumors compared with tumors from the monotherapy groups. Taken together, these results suggest that the combined administration of oAd/IL12/ GM-RLX and $\alpha$ PD-1 resulted in the attraction of more activated $\mathrm{T}$ cells and facilitated their infiltration into poorly immunogenic tumors.

To assess cancer-specific immune responses, DLN-based immune cells from hamsters treated with $\alpha \mathrm{PD}-1$, oAd/ IL12/GM-RLX, or oAd/IL12/GM-RLX plus $\alpha$ PD-1 were harvested and cocultured with irradiated HaP-T1 cancer cells. After 3 days, the supernatant was harvested, and the levels of IFN- $\gamma$ secreted by cancer-specific lymphocytes were measured by ELISA. As shown in figure $6 \mathrm{E}$, IFN- $\gamma$ expression was not detected in the $\alpha \mathrm{PD}-1$-treated group, suggesting that ICI alone cannot sufficiently induce antitumor immune responses in poorly immunogenic tumors. In marked contrast, both oAd/IL12/GM-RLX monotherapy and combination therapy with ICI resulted in a markedly elevated IFN- $\gamma$ expression level, which was 2.2-fold higher in the combination therapy group versus the monotherapy group $(* * * \mathrm{p}<0.001)$. Together, these results suggest that immune stimulatory oncolytic Ad can induce tumor-specific immune responses in 'cold' tumors and that this effect can further be enhanced by concomitant ICI administration.

\section{Overcoming the ICI resistance of poorly immunogenic tumors with oAd/IL12/GM-RLX}

A large subset of patients receives no clinical benefits from ICIs and tumor hyperprogression has been reported in some cases. ${ }^{28}$ To explore whether subsequent administration of oAd/IL12/GM-RLX provides therapeutic benefits in such ICI refractory subsets, the inhibitory effect of oAd/IL12/GM-RLX on tumor growth was assessed in tumors pretreated with ICI therapy. oAd/IL12/GM-RLX was administered four times at 2-day intervals when tumor growth was no longer inhibited by $\alpha \mathrm{PD}-1$ monotherapy ( 9 days postinitial treatment as determined in figure $4 \mathrm{~A}$ ). As shown in figure $7 \mathrm{~A}, \alpha \mathrm{PD}-1$ as monotherapy failed to suppress the tumor growth, although the tumor volume was steady for about 4 days after administration (tumor growth rates: $29.4 \mathrm{~mm}^{3} /$ day during days $8-12$ vs $144.2 \mathrm{~mm}^{3}$ /day during days 12-28). In sharp contrast, the administration of oAd/IL12/GM-RLX following $\alpha$ PD-1 treatment continued to suppress tumor growth during both early and late time intervals (tumor growth rates: $9.8 \mathrm{~mm}^{3}$ / day during days $9-17$ vs $22.6 \mathrm{~mm}^{3}$ /day during 

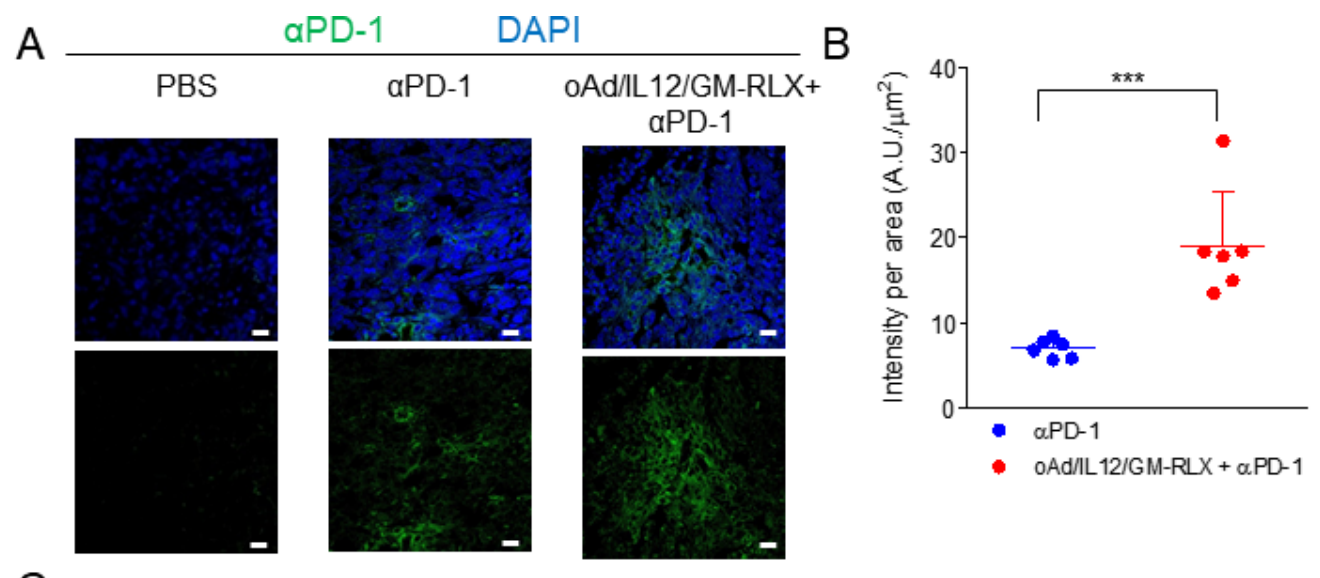

C
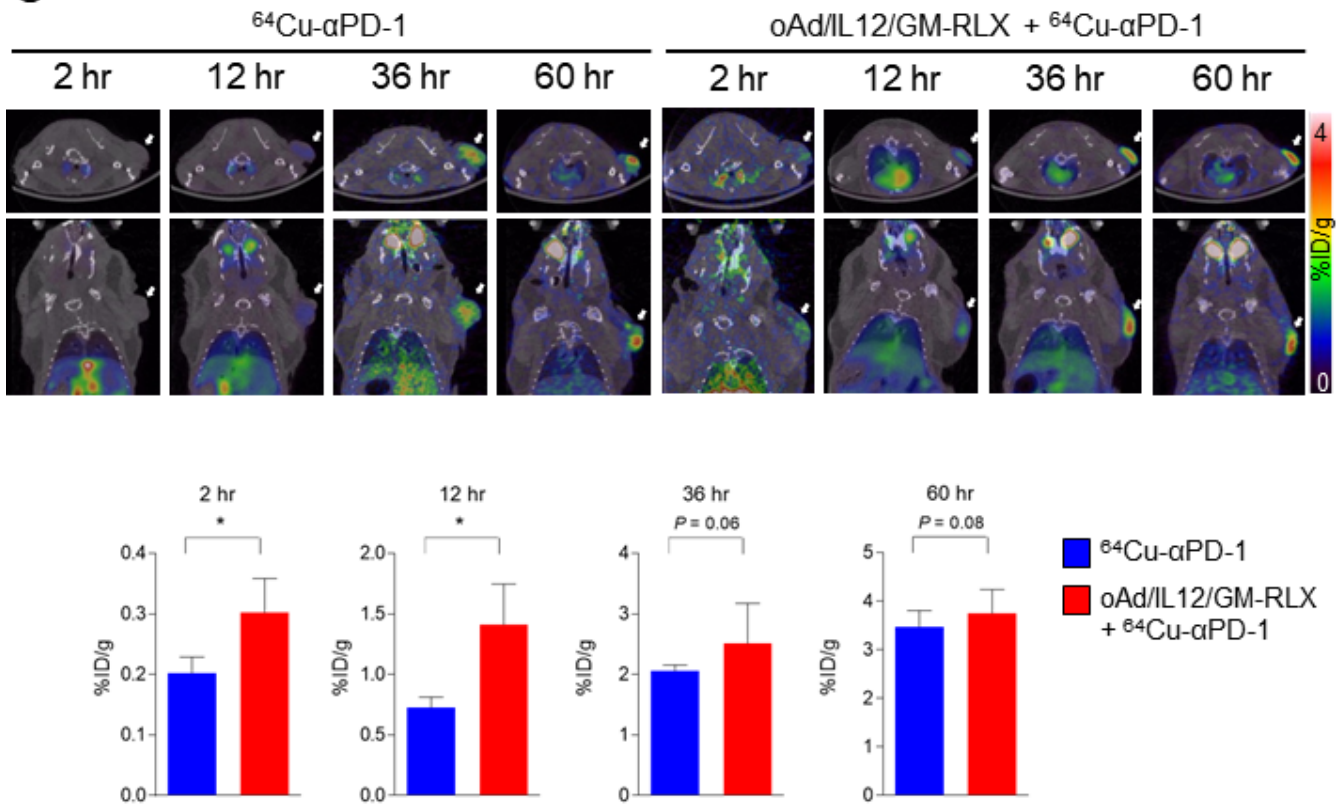

${ }^{64} \mathrm{Cu}-\mathrm{aPD}-1$

$\mathrm{oAd} / \mathrm{LL} 12 / \mathrm{GM}-\mathrm{RLX}$

$+{ }^{64} \mathrm{Cu}-\mathrm{aPD}-1$

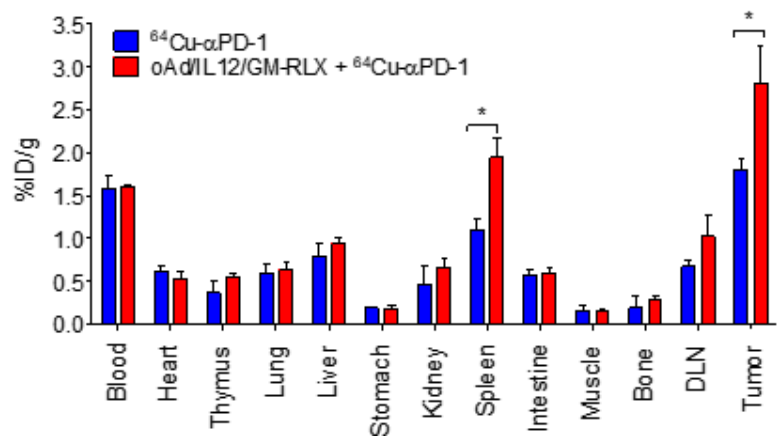

Figure 5 Distribution of $\alpha$ PD-1 Ab in HaP-T1 tumors. (A) Fluorescence images of 488- $\alpha$ PD-1 ab in HaP-T1 tumors. Tumors were isolated at 5 days after the final injection of PBS, 488- $\alpha$ PD-1, or oAd/IL12/GM-RLX plus 488- $\alpha$ PD-1. 488-TZB (green)stained and DAPI-stained nuclei (blue) are shown for each group. The scale bar represents $20 \mu \mathrm{m}$. (B) Quantitative analysis of the accumulation of $\alpha P D-1$ in tumor sections. The intensity of $488-\alpha P D-1$ and the tumor slice areas were measured in the fluorescence images. Data are presented as mean $\pm S D(n=5) ; \alpha P D-1$ versus oAd/IL12/GM-RLX $+\alpha P D-1,{ }^{\star * *} p<0.001$. Statistical analyses were performed by two-tailed Student t-test. (C) ImmunoPET images of subcutaneous HaP-T1 tumor-bearing hamsters. PET images were acquired for $15 \mathrm{~min}$ at 2, 12, and 36 hours after injection of ${ }^{64} \mathrm{Cu}-\alpha \mathrm{PD}-1 \mathrm{AB}(17.39-20.35 \mathrm{MBq}$, $400 \mu \mathrm{g}$ of $\alpha \mathrm{PD}-1)$. Tumors are indicated with the white arrows. Radioactivity in tissues are represented as $\% \mathrm{ID} / \mathrm{g} \pm \mathrm{SD}(\mathrm{n}=3)$; ${ }^{64} \mathrm{Cu}-\alpha \mathrm{PD}-1$ versus oAd/IL12/GM-RLX $+{ }^{64} \mathrm{Cu}-\alpha \mathrm{PD}-1,{ }^{*} \mathrm{p}<0.05$. Statistical analyses were performed by two-tailed Student $\mathrm{t}-$ test. (D) Biodistribution study after injection of ${ }^{64} \mathrm{Cu}-\alpha \mathrm{PD}-1$ at 60 hours. The organs and tumors were isolated from hamsters and the radioactivity was measured. Radioactivity in tissues is represented as $\%$ ID/g $\pm S D(n=3) ;{ }^{64} \mathrm{Cu}-\alpha \mathrm{PD}-1$ versus oAd/IL12/ $\mathrm{GM}-\mathrm{RLX}+{ }^{64} \mathrm{Cu}-\alpha \mathrm{PD}-1,{ }^{*} \mathrm{p}<0.05$. Statistical analyses were performed by two-tailed Student t-test. $\alpha \mathrm{PD}-1$, anti-programmed cell death protein 1; Ab, antibody; DAPI, 4,6-diamidino-2-phenyindole; PBS, phosphate-buffered saline; DLN, draining lymph node; PET, positron emission tomography. 


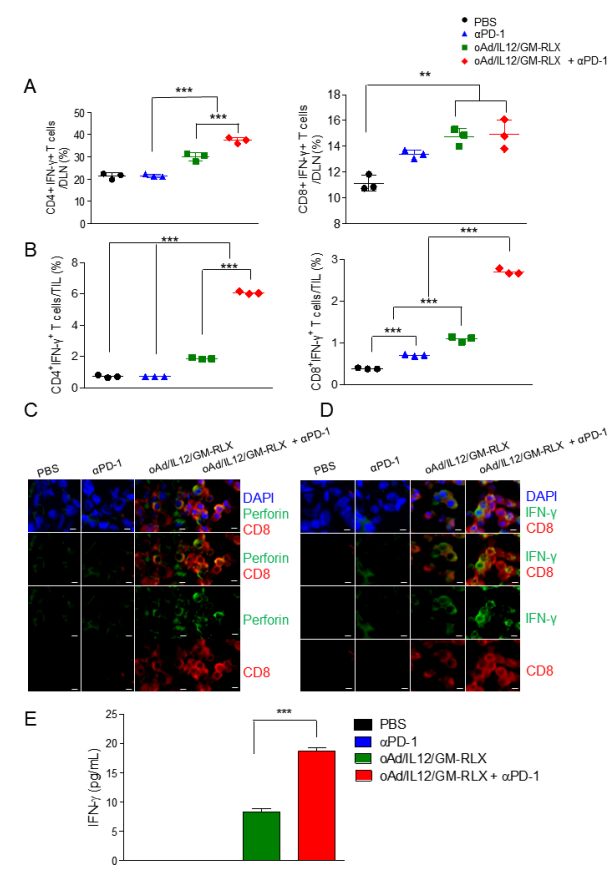

Figure 6 Analysis of activated T cells in DLN and tumor tissue. HaP-T1 tumor-bearing hamsters were injected with PBS, $\alpha$ PD-1 $(10 \mathrm{mg} / \mathrm{kg})$, oAd/IL12/GM-RLX $\left(7 \times 10^{7}\right.$

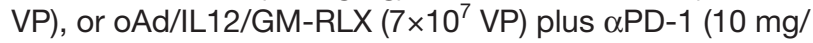
$\mathrm{kg})$. Lymphocytes were harvested 12 days after the initial treatment to perform FACS analysis for $\mathrm{CD}^{+} \mathrm{IFN}-\gamma^{+}$or $\mathrm{CD}^{+}{ }^{+} \mathrm{IFN}-\gamma^{+}$T cells in (A) DLN or (B) tumor tissue. Data are presented as mean $\pm \mathrm{SD}$ of three independent measurements; ${ }^{* *} p<0.01,{ }^{* * *} p<0.001$. (C,D) Double-immunofluorescence staining for colocalization of CD8 (red) and perforin or IFN- $\gamma$ (green) in tumor tissues treated with PBS, oAd/IL12/GM-RLX, $\alpha P D-1$, or oAd/IL12/GM-RLX plus $\alpha$ PD-1 and isolated at 5 days after the final treatment. The scale bar represents 5 $\mu \mathrm{m}$. (E) Lymphocytes were collected from hamster DLNs at day 12 after the initial virus injection and coincubated with preirradiated HaP-T1 cells. After 3 days of coincubation, an IFN- $\gamma$ ELISA was carried out. Data are presented as mean $\pm S D(n=3)$; oAd/IL12/GM-RLX versus oAd/IL12/GM$R L X+\alpha P D-1,{ }^{* *} p<0.001$. Statistical analyses were performed by one-way analysis of variance. PBS, phosphate-buffered saline; $\alpha P D-1$, anti-programmed cell death protein 1; DAPI, 4,6-diamidino-2-phenyindole; DLN, draining lymph node; FACS, fluorescence-activated cell sorting; IFN, interferon.

days 17-30) more effectively compared with $\alpha$ PD-1 monotherapy, suggesting that oncolytic Ad can effectively treat ICI refractory tumors of high tumor burden. On day 25 postadministration, oAd/IL12/GM-RLX in $\alpha$ PD-1 resistant tumor $(\alpha \mathrm{PD}-1+\mathrm{oAd} / \mathrm{IL} 12 / \mathrm{GM}-\mathrm{RLX})$ still exhibited most potent tumor growth inhibition when compared with PBS and $\alpha$ PD-1 treatment, showing that oAd/ IL12/GM-RLX can suppress tumor growth effectively in hamsters with ICI refractory tumors of high tumor burden at the time of virus injection: specifically, $\alpha$ PD- 1 Ab treatment followed by oAd/IL12/GM-RLX yielded a $62.5 \%$ and $49.3 \%$ lower tumor burden than did $\alpha \mathrm{PD}-1$ or oAd/ IL12/GM-RLX treatment alone, respectively (figure 7B, $* * * \mathrm{p}<0.001)$. Taken together, these results suggest that administration of oAd/IL12/GM-RLX to ICI refractory
A

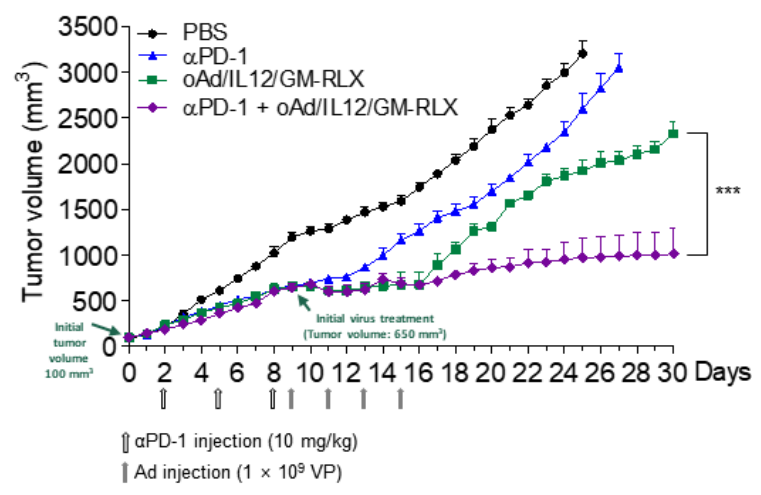

B

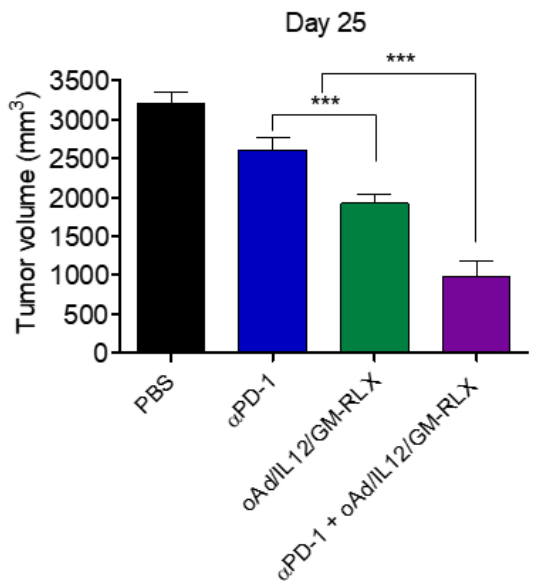

Figure 7 Overcoming $\alpha$ PD-1 resistance with oAd/IL12/ GM-RLX. (A) Syrian hamsters were subcutaneously injected with HaP-T1 cells to establish pancreatic tumors. When the average tumor volume reached $90-100 \mathrm{~mm}^{3}$, the hamsters were injected with $10 \mathrm{mg} / \mathrm{kg}$ of $\alpha \mathrm{PD}-1$ intraperitoneally (days 2, 5, and 8; empty arrows) and $1 \times 10^{9} \mathrm{VP}$ of oAd/IL12/GMRLX intratumorally (days $9,11,13$, and 15 ; gray arrows). The tumor volume was measured every day until the end of the study. Data are presented as mean $\pm S D(n=5) ; o A d / I L 12 / G M-$ $R L X$ versus $\alpha P D-1+o A d / I L 12 / G M-R L X,{ }^{* * *} p<0.001$. Statistical analyses were performed by one-way ANOVA. (B) Bar graph of the tumor size at day 25. The data were based on (A). Data are presented as mean $\pm S D(n=5) ; O A d / I L 12 / G M-R L X$ versus $\alpha P D-1$ or $\alpha P D-1+o A d / I L 12 / G M-R L X,{ }^{* \star *} p<0.001$. Statistical analyses were performed by one-way ANOVA. PBS, phosphate-buffered saline; $\alpha \mathrm{PD}-1$, anti-programmed cell death protein 1; Ad, adenovirus; ANOVA, analysis of variance; VP, viral particle.

tumors could induce durable tumor growth suppression and be a beneficial therapeutic regimen for patients who are resistant to ICI monotherapy.

\section{DISCUSSION}

A hostile tumor microenvironment remains a critical challenge to achieving a high therapeutic index with cancer therapies. Several factors in the tumor environment, such as dense ECM, poor vascular permeability, hypoxia, and 
poor lymphatic drainage, restrict intratumoral infiltration and dispersion of drugs and therapeutic cells. ${ }^{3}{ }^{29}$ Recently, there was a report that the efficacy of immune check point blockade could be enhanced using intratumorally injected GM-CSF armed oncolytic Ad. ${ }^{30}$ However, in the solid tumor, overcoming physical barrier as well as physiological barrier would be important. Previously, we showed RLX could degrade the ECM within the tumor. $^{13} 20$ Similarly, RLX conjugated superparamagnetic iron oxide nanoparticles showed the degradation of fibrosis in pancreatic cancer, inducing enhancement of efficacy of gemcitabine. ${ }^{31}$

In our present study, to overcome the physical barrier of solid tumor, oncolytic Ad expressing RLX was used in combination with therapeutic Abs (TZB and $\alpha \mathrm{PD}-1$ ) and radiolabeled Abs $\left({ }^{64} \mathrm{Cu}-\mathrm{TZB}\right.$ and $\left.{ }^{64} \mathrm{Cu}-\alpha \mathrm{PD}-1\right)$ in the present report to promote drug penetration through degradation of the tumor ECM. Moreover, to overcome the immunological barrier of solid tumor, we used 'armed oncolytic Ad' (oAd/IL12/GM-RLX) for the treatment of pancreatic tumors that are refractory to ICI. Of note, oAd/IL12/GM-RLX coexpresses genes encoding four proteins, IL-12 consisting of p35 and p40 subunits, GM-CSF and RLX. This is the first report demonstrating that four genes (IL-12p35, IL-12p40, GM-CSF, and RLX) can be effectively transferred by a single viral vector. oAd/ IL12/GM-RLX showed a more potent antitumor effect than did previously reported oAds at a dose that is at least 10-fold lower. ${ }^{32}$ Furthermore, oAd/IL12/GM-RLX remodeled the physical and immunological barriers in poorly immunogenic solid tumors, leading to enhanced potency of ICI.

TZB is a monoclonal Ab specific to human epidermal growth factor receptor 2 (Her2) that induces its therapeutic effects in Her2-positive cancer by directing Ab-dependent cell-mediated cytotoxicity. ${ }^{33}$ However, the therapeutic efficacy of such Abs is limited because of insufficient penetration into solid tumors. Only $0.001 \%-$ $0.01 \%$ of the injected therapeutic Abs reach the tumor of patients. ${ }^{34} 35$ To overcome this limitation, researchers have manipulated the size, charge, and antigen-binding affinity of Abs to increase their penetration and dispersion. ${ }^{36}$ Alternatively, therapeutic Abs have been coadministered with other substances to increase vascular permeability or reduce epithelial barriers. ${ }^{37} 38$ In the present study, we used an RLX-expressing oncolytic Ad to increase intratumoral Ab accumulation through degradation of the ECM.

Indeed, oAd/RLX was able to effectively degrade aberrant tumor ECM (online supplementary figure S1C), thus resulting in significantly enhanced penetration and dispersion of TZB (figure 2A-C). Of note, TZB penetrated significantly more into the center of the tumor tissue when coadministered with oAd/RLX, even though the vascular density was not altered (online supplementary figure S3). In addition, ECM reduction by RLX increased the sensitivity of PET imaging by enhancing tumor uptake of radiolabeled Abs (figure 3). These results were likely achieved by attenuation of interstitial pressure following epithelial cell junction opening, RLX-mediated degradation of the ECM, and oncolysis of tumor, ultimately relieving mechanical constraints on the tumor tissue and creating more interstitial space for drugs to maneuver. ${ }^{39}$ Furthermore, oncolytic Ad-mediated expression of RLX has been reported to promote apoptotic tumor cell death, ${ }^{13}$ which in theory could also lower the interstitial pressure in solid tumors. ${ }^{40}$ Our strategies enhancing tumor uptake of radiolabeled-Abs are expected to be also useful for $\alpha$-radioimmunotherapy if ${ }^{64} \mathrm{Cu}$ is changed to $\alpha$-particle, such as ${ }^{211} \mathrm{At}\left(\mathrm{t}_{1 / 2}, 7.2\right.$ hours $)$ and ${ }^{213} \mathrm{Bi}\left(\mathrm{t}_{1 / 2}, 46 \mathrm{~min}\right)$.

Among various cancer-targeted therapeutic Abs used in the clinic, ICIs are unique in their immune modulatory ability, which allows the host immune system to overcome tumor-induced immunosuppression and immune escape mechanisms to mount a systemic antitumor immune response against cancer, yielding remarkable achievements in clinical trials involving patients with cancers originating from various tissues. ${ }^{41}$ However, ICI monotherapy elicits insufficient antitumor efficacy in some patients due to various immune escape mechanisms of tumors. ${ }^{42}$ Particularly, the tumor microenvironment functions as a limiting factor for ICIs, as it does for other chemotherapeutics for which penetration of immune cells is hindered by physical barriers such as tight epithelial junctions and a dense layer of tumor ECM. 'Cold' tumors, which are refractory to ICIs, often possess a dense ECM, resulting in poor penetration of activated immune cells in a large subset of patients, ${ }^{43}$ with only $20 \%-30 \%$ benefiting from ICI therapy. ${ }^{44}$ Among cold tumors, pancreatic cancer is particularly problematic due to a strong desmoplastic reaction leading to the formation of dense tumor ECM. ${ }^{45}$

On the basis of these reports, we hypothesized that an RLX-expressing oncolytic Ad may enhance the efficacy of ICIs through ECM degradation. Indeed, the degradation of tumor ECM induced by oAd/IL12/GM-RLX led to enhanced ICI penetration, similar to what was observed with the combination of oAd/RLX and TZB as shown in figures 1-3 and efficient infiltration of activated $\mathrm{T}$ cells in highly desmoplastic pancreatic tumor tissues (figures 5 and 6). These attributes are highly favorable for the treatment of pancreatic cancer, as patients with pancreatic cancer with high density and quantity of TIL have been shown to exhibit higher overall survival rate and progression-free survival than those with low TIL count. ${ }^{46}$ In line with our findings, a phase II clinical trial, which combines IL-12-expressing plasmid DNA and $\alpha$ PD-1 Ab, demonstrated that IL-12 expression enhances the response to $\alpha \mathrm{PD}-1$ therapy in patients with low levels of TILs. ${ }^{47}$ Because viral vectors, especially Ad, often express therapeutic genes at a much higher level than plasmid vectors (the difference is further magnified when oncolytic vectors are compared due to the exponential amplification in therapeutic gene expression following viral replication), immune stimulatory oncolytic viral vectors in combination with 
ICIs may yield promising results against cold tumors in future clinical trials.

Another major limitation of ICI therapy is disease relapse, and recurrence among responding patients as drug-induced selective pressure leads to the survival of tumor cells with adaptive resistance against ICIs. Tumor cells frequently escape ICI-mediated immune modulation by upregulation of an alternative immune checkpoint. ${ }^{48}$ Currently, various coadministration strategies are being evaluated and have demonstrated promising results in clinical trials. ${ }^{49}$ In line with these trends, combined administration of an ICI and oAd/IL12/GM-RLX for the treatment of pancreatic tumors that are refractory to ICI led to durable and potent tumor growth suppression, whereas either immunotherapeutic alone eventually resulted in tumor progression (figure 7), demonstrating that oAd/ IL12/GM-RLX could convert ICI refractory cold tumors to 'hot' tumors, thus overcoming the poor efficacy of ICI monotherapy against non-immunogenic tumors.

Our initial assessment of combination therapy using TBZ and oAd/RLX via systemic administration was performed in a human tumor xenograft model using immunodeficient nude mice, focusing on whether RLX degrades dense tumor ECM originating from human cancer. Following our initial assessment clearly illustrating that oAd/RLX-mediated degradation of tumor ECM can improve TBZ penetration and accumulation in tumor tissues, we hypothesized that similar enhancement of efficacy can be achieved by combining RLX-expressing oAd with other Ab therapeutics. To evaluate our second assertion, immunocompetent subcutaneous or orthotopic pancreatic tumor models in Syrian hamsters were used to ascertain that oAd/IL12/GM-RLX in combination with $\alpha \mathrm{PD}-1$ enhances ICI distribution in tumor tissues, as well as promotes intratumoral infiltration and activation of $\mathrm{T}$ cells. An orthotopic pancreatic tumor model in Syrian hamster was reported to closely recapitulate morphological and biological aspects of pancreatic cancer in human. ${ }^{25}$ For the study in an immune-competent host, intratumoral injection was used due to the technical hurdles toward intravenous administration in hamsters where tail vein is unavailable. Use of femoral vein was also not suitable for long-term follow-up ( $\sim 30$ days). Despite intravenous injection being preferred route of injection over local administration methods, intratumoral or intralesional injection methods were still widely used in clinics. $^{50}$

In sum, an RLX-expressing oncolytic Ad can greatly enhance $\mathrm{Ab}$ penetration and distribution by degrading and remodeling the tumor ECM, demonstrated in both subcutaneous and orthotropic tumor model, leading to potent combination therapy effect against both primary and metastatic tumors. Further, concomitant expression of two immune stimulatory cytokines (IL-12 and GM-CSF) and RLX by a single oncolytic Ad vector led to a significant improvement in tumor growth inhibition. Prominently, a combination of oAd/IL12/GM-RLX and $\alpha \mathrm{PD}-1 \mathrm{Ab}$ improved the accumulation of activated $\mathrm{T}$ cells in desmoplastic tumors, which was achieved by increasing the intratumoral penetration of the Ab, antitumor cytokine expression, and ECM degradation. Collectively, a combination of oncolytic Ad coexpressing immune stimulatory and ECM degrading factors with $\alpha \mathrm{PD}-1$ induced durable tumor growth suppression of ICI refractory cold tumors in high tumor burden scenarios by favorably modulating both physical and immunological aspects of poorly immunogenic solid tumor.

\section{Author affiliations}

${ }^{1}$ Department of Bioengineering, College of Engineering, Hanyang University, Seoul, Korea (the Republic of)

${ }^{2}$ Division of RI Application, Korea Institute of Radiological and Medical Sciences, Seoul, Korea (the Republic of)

${ }^{3}$ Department of Research and Development, GeneMedicine Co., Ltd, Seoul, Korea (the Republic of)

${ }^{4}$ Radiological and Medico-Oncological Sciences, University of science and technology (UST), Seoul, Korea (the Republic of)

${ }^{5}$ Institute of Nano Science and Technology (INST), Hanyang University, Seoul, Korea (the Republic of)

Acknowledgements We thank Hyun Park and Jung Young Kim for providing ${ }^{64} \mathrm{Cu}$ and Seonhwa Lee for dosimetry analysis. ${ }^{64} \mathrm{Cu}$ was produced by the $50-\mathrm{MeV}$ cyclotron at KIRAMS.

Contributors C-OY, JSK, B-KJ and HYK designed the research studies. B-KJ, HYK, HKa, HMA and YN conducted the experiments. B-KJ, HYK, HKa, HMA, JWH, YN, $\mathrm{HKi}$, JSK and C-OY analyzed the data. C-OY, JSK, B-KJ, HYK and JWH wrote the manuscript. All authors reviewed the results and approved the final version of the manuscript.

Funding This work was supported by grants from the National Research Foundation of Korea (2016M3A9B5942352, C-OY), KIRAMS (505362020 and 50461-2020) and the research fund of Hanyang University (HY-2011-G-201100000001880, C-OY).

Competing interests JWH and HMA are employee of GeneMedicine. C-OY is the CEO in GeneMedicine. The remaining authors have no conflicts of interest to declare.

Patient consent for publication Not required.

Ethics approval All mice-related experiments and positron emission tomography imaging were performed under a protocol approved by institutional animal care and use committee (IACUC) of the Korea Institute of Radiological and Medical Sciences (KIRAMS 2016-0020 and KIRAMS 2018-0016). All Syrian hamster-related experiments were conducted under the institutional guidelines established by IACUC of Hanyang University (2015-0233A and 2017-0240A).

Provenance and peer review Not commissioned; externally peer reviewed.

Data availability statement All data generated or analyzed during this work are included either in this article or in the supplementary information files.

Open access This is an open access article distributed in accordance with the Creative Commons Attribution 4.0 Unported (CC BY 4.0) license, which permits others to copy, redistribute, remix, transform and build upon this work for any purpose, provided the original work is properly cited, a link to the licence is given, and indication of whether changes were made. See https://creativecommons.org/ licenses/by/4.0/.

\section{ORCID iDs}

Hyeongi Kim http://orcid.org/0000-0001-6990-3900

Chae-0k Yun http://orcid.org/0000-0002-9466-4531

\section{REFERENCES}

1 Kaplon H, Muralidharan M, Schneider Z. Reichert JM: antibodies to watch in 2020. MAbs 2020;12:1703531.

2 Scott AM, Lee F-T, Tebbutt N, et al. A phase I clinical trial with monoclonal antibody ch806 targeting transitional state and mutant 
epidermal growth factor receptors. Proc Natl Acad Sci U S A 2007;104:4071-6.

3 Choi I-K, Strauss R, Richter M, et al. Strategies to increase drug penetration in solid tumors. Front Oncol 2013;3:193.

4 Egeblad M, Rasch MG, Weaver VM. Dynamic interplay between the collagen scaffold and tumor evolution. Curr Opin Cell Biol 2010;22:697-706.

5 Choi J-W, Lee J-S, Kim SW, et al. Evolution of oncolytic adenovirus for cancer treatment. Adv Drug Deliv Rev 2012;64:720-9.

6 Hong J, Yun C-O. Relaxin gene therapy: a promising new treatment option for various diseases with aberrant fibrosis or irregular angiogenesis. Mol Cell Endocrinol 2019;487:80-4.

7 Topalian SL, Taube JM, Anders RA, et al. Mechanism-Driven biomarkers to guide immune checkpoint blockade in cancer therapy. Nat Rev Cancer 2016;16:275-87.

8 Spranger S. Mechanisms of tumor escape in the context of the T-cell-inflamed and the non-T-cell-inflamed tumor microenvironment. Int Immunol 2016;28:383-91.

9 Okazaki T, Chikuma S, Iwai Y, et al. A rheostat for immune responses: the unique properties of PD-1 and their advantages for clinical application. Nat Immunol 2013;14:1212-8.

10 llett E, Kottke T, Thompson J, et al. Prime-Boost using separate oncolytic viruses in combination with checkpoint blockade improves anti-tumour therapy. Gene Ther 2017;24:21-30.

11 Bai Y, Hui P, Du X, et al. Updates to the antitumor mechanism of oncolytic virus. Thorac Cancer 2019;10:1031-5.

12 Guo S, Contratto M, Miller G, et al. Immunotherapy in pancreatic cancer: Unleash its potential through novel combinations. World $\mathrm{J}$ Clin Oncol 2017;8:230

$13 \mathrm{Kim} \mathrm{J-H}$, Lee Y-S, Kim H, et al. Relaxin expression from tumortargeting adenoviruses and its intratumoral spread, apoptosis induction, and efficacy. J Natl Cancer Inst 2006;98:1482-93.

14 Huang J-H, Zhang S-N, Choi K-J, et al. Therapeutic and tumorspecific immunity induced by combination of dendritic cells and oncolytic adenovirus expressing IL-12 and 4-1BBL. Mol Ther 2010;18:264-74.

15 Wang S, Shin IS, Hancock H, et al. Pulsed high intensity focused ultrasound increases penetration and therapeutic efficacy of monoclonal antibodies in murine xenograft tumors. J Control Release 2012;162:218-24.

$16 \mathrm{Na} \mathrm{Y,} \mathrm{Choi} \mathrm{J-W,} \mathrm{Kasala} \mathrm{D,} \mathrm{et} \mathrm{al.} \mathrm{Potent} \mathrm{antitumor} \mathrm{effect} \mathrm{of}$ neurotensin receptor-targeted oncolytic adenovirus co-expressing decorin and Wnt antagonist in an orthotopic pancreatic tumor model. $J$ Control Release 2015;220:766-82.

17 van Dongen GAMS, Visser GWM, Lub-de Hooge MN, et al. Immuno-PET: a navigator in monoclonal antibody development and applications. Oncologist 2007;12:1379-89.

18 Kim JY, Park H, Lee JC, et al. A simple Cu-64 production and its application of Cu-64 ATSM. Appl Radiat Isot 2009;67:1190-4.

19 Choi I-K, Li Y, Oh E, et al. Oncolytic adenovirus expressing IL-23 and p35 elicits IFN- $\gamma$ - and TNF- $\alpha$-co-producing T cell-mediated antitumor immunity. PLoS One 2013;8:e67512.

20 Jung KH, Choi I-K, Lee H-S, et al. Oncolytic adenovirus expressing relaxin (YDC002) enhances therapeutic efficacy of gemcitabine against pancreatic cancer. Cancer Lett 2017;396:155-66.

21 Choi K-J, Zhang S-N, Choi I-K, et al. Strengthening of antitumor immune memory and prevention of thymic atrophy mediated by adenovirus expressing IL-12 and GM-CSF. Gene Ther 2012;19:711-23.

22 Heatley JJ, Harris MC. Chapter 15 - Hamsters and Gerbils. In: Mitchell MA, Tully TN, eds. Manual of exotic PET practice. Saint Louis: W.B. Saunders, 2009: 406-32.

23 Sonabend AM, Ulasov IV, Han Y, Rolle CE, et al. Biodistribution of an oncolytic adenovirus after intracranial injection in permissive animals: a comparative study of Syrian hamsters and cotton rats. Cancer Gene Ther 2009:16:362-72.

24 Abbasi Oshaghi E, Khodadadi I, Saidijam M, et al. Lipid lowering effects of hydroalcoholic extract of Anethum graveolens $\mathrm{L}$. and dill tablet in high cholesterol fed hamsters. Cholesterol 2015;2015:1-7.

25 Suklabaidya S, Das B, Ali SA, et al. Characterization and use of HapT1-derived homologous tumors as a preclinical model to evaluate therapeutic efficacy of drugs against pancreatic tumor desmoplasia. Oncotarget 2016;7:41825-42.

26 Hicks AM, Chou J, Capanu M, et al. Pancreas adenocarcinoma: ascites, clinical manifestations, and management implications. Clin Colorectal Cancer 2016;15:360-8.
27 Oelkrug C, Ramage JM. Enhancement of T cell recruitment and infiltration into tumours. Clin Exp Immunol 2014;178:1-8.

28 Champiat S, Dercle L, Ammari S, et al. Hyperprogressive disease is a new pattern of progression in cancer patients treated by anti-PD-1/ PD-L1. Clin Cancer Res 2017;23:1920-8.

29 Sriraman SK, Aryasomayajula B, Torchilin VP. Barriers to drug delivery in solid tumors. Tissue Barriers 2014;2:e29528.

30 Chon HJ, Lee WS, Yang H, et al. Tumor microenvironment remodeling by intratumoral oncolytic vaccinia virus enhances the efficacy of Immune-Checkpoint blockade. Clin Cancer Res 2019;25:1612-23.

31 Mardhian DF, Storm G, Bansal R, et al. Nano-targeted relaxin impairs fibrosis and tumor growth in pancreatic cancer and improves the efficacy of gemcitabine in vivo. $J$ Control Release 2018;290:1-10.

32 Oh E, Choi I-K, Hong J, et al. Oncolytic adenovirus coexpressing interleukin-12 and decorin overcomes Treg-mediated immunosuppression inducing potent antitumor effects in a weakly immunogenic tumor model. Oncotarget 2017;8:4730-46.

33 Luque-Cabal M, García-Teijido P, Fernández-Pérez Y, et al. Mechanisms behind the resistance to trastuzumab in HER2-amplified breast cancer and strategies to overcome it. Clin Med Insights Oncol 2016;10:21-30.

34 Christiansen J, Rajasekaran AK. Biological impediments to monoclonal antibody-based cancer immunotherapy. Mol Cancer Ther 2004;3:1493-501.

35 Epenetos AA, Snook D, Durbin $\mathrm{H}$, et al. Limitations of radiolabeled monoclonal antibodies for localization of human neoplasms. Cancer Res 1986;46:3183-91.

36 Beckman RA, Weiner LM, Davis HM. Antibody constructs in cancer therapy: protein engineering strategies to improve exposure in solid tumors. Cancer 2007;109:170-9.

37 Beyer I, van Rensburg R, Strauss R, et al. Epithelial junction opener JO-1 improves monoclonal antibody therapy of cancer. Cancer Res 2011;71:7080-90.

38 Liu Y, Ji M, Wong MK, et al. Enhanced therapeutic efficacy of iRGD-conjugated crosslinked multilayer liposomes for drug delivery. Biomed Res Int 2013;2013:378380.

39 Ariffin $\mathrm{AB}$, Forde PF, Jahangeer $\mathrm{S}$, et al. Releasing pressure in tumors: what do we know so far and where do we go from here? A review. Cancer Res 2014;74:2655-62.

40 Griffon-Etienne G, Boucher Y, Brekken C, et al. Taxane-induced apoptosis decompresses blood vessels and lowers interstitial fluid pressure in solid tumors: clinical implications. Cancer Res 1999;59:3776-82.

41 Nishino M, Ramaiya NH, Hatabu $\mathrm{H}$, et al. Monitoring immunecheckpoint blockade: response evaluation and biomarker development. Nat Rev Clin Oncol 2017;14:655-68.

42 O'Donnell JS, Smyth MJ, Teng MWL. Acquired resistance to antiPD1 therapy: checkmate to checkpoint blockade? Genome Med 2016;8:111.

43 Stanton SE, Disis ML. Clinical significance of tumor-infiltrating lymphocytes in breast cancer. J Immunother Cancer 2016;4:59.

44 Winograd R, Byrne KT, Evans RA, et al. Induction of T-cell immunity overcomes complete resistance to PD- 1 and CTLA- 4 blockade and improves survival in pancreatic carcinoma. Cancer Immunol Res 2015;3:399-411.

45 Vonderheide RH, Bayne LJ. Inflammatory networks and immune surveillance of pancreatic carcinoma. Curr Opin Immunol 2013;25:200-5.

46 Miksch RC, Schoenberg MB, Weniger M, et al. Prognostic impact of tumor-infiltrating lymphocytes and neutrophils on survival of patients with upfront resection of pancreatic cancer. Cancers 2019;11:39.

47 Algazi A, Tsai KK, Takamura KT, et al. Le MH: Abstract CT134: intratumoral electroporation of plasmid IL-12 can prime response to anti-PD1/PD-L1 blockade in patients with stage III/IV-M1a melanoma. AACR 2016.

48 Koyama S, Akbay EA, Li YY, et al. Adaptive resistance to therapeutic PD-1 blockade is associated with upregulation of alternative immune checkpoints. Nat Commun 2016;7:10501.

49 Wong RM. Cameron RB: Immune Checkpoint Blockade and Adaptive Immune Resistance in Cancer. In Immunotherapy-Myths, Reality, Ideas, Future. InTech 2017.

50 Aznar MA, Tinari N, Rullán AJ, et al. Intratumoral delivery of Immunotherapy-Act locally, think globally. J Immunol 2017;198:31-9. 